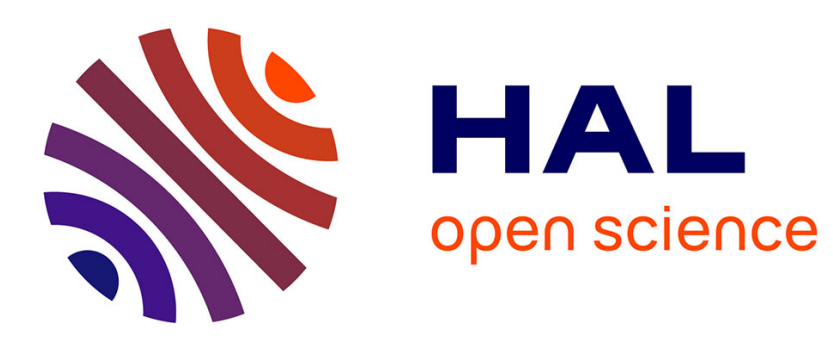

\title{
A robust super-resolution approach with sparsity constraint in acoustic imaging
}

Ning Chu, José Picheral, Ali Mohammad-Djafari, Nicolas Gac

\section{To cite this version:}

Ning Chu, José Picheral, Ali Mohammad-Djafari, Nicolas Gac. A robust super-resolution approach with sparsity constraint in acoustic imaging. Applied Acoustics, 2014, 76, pp.197-208. 10.1016/j.apacoust.2013.08.007 . hal-00794236v8

\section{HAL Id: hal-00794236 \\ https://hal.science/hal-00794236v8}

Submitted on 29 Aug 2013

HAL is a multi-disciplinary open access archive for the deposit and dissemination of scientific research documents, whether they are published or not. The documents may come from teaching and research institutions in France or abroad, or from public or private research centers.
L'archive ouverte pluridisciplinaire HAL, est destinée au dépôt et à la diffusion de documents scientifiques de niveau recherche, publiés ou non, émanant des établissements d'enseignement et de recherche français ou étrangers, des laboratoires publics ou privés. 


\title{
A robust super-resolution approach with sparsity constraint in acoustic imaging th
}

\author{
Ning CHU ${ }^{a, 1, *}$, José PICHERAL ${ }^{\mathrm{b}}$, Ali MOHAMMAD-DJAFARI ${ }^{\mathrm{a}}$, Nicolas GAC ${ }^{\mathrm{a}}$ \\ ${ }^{a}$ Laboratoire des signaux et systèmes (L2S), CNRS-SUPELEC-UNIV PARIS SUD, 91192 GIF-SUR-YVETTE, FRANCE \\ ${ }^{b}$ SUPELEC, Département du Signal et Systèmes Electroniques, 91192 GIF-SUR-YVETTE, FRANCE
}

\begin{abstract}
Acoustic imaging is a standard technique for mapping acoustic source powers and positions from limited observations on microphone sensors, which often causes an ill-conditioned inverse problem. In this article, we firstly improve the forward model of acoustic power propagation by considering background noises at the sensor array, and the propagation uncertainty caused by wind tunnel effects. We then propose a robust super-resolution approach via sparsity constraint for acoustic imaging in strong background noises. The sparsity parameter is adaptively derived from the sparse distribution of source powers. The proposed approach can jointly reconstruct source powers and positions, as well as the background noise power. Our approach is compared with the conventional beamforming, deconvolution and sparse regularization methods by simulated, wind tunnel data and hybrid data respectively. It is feasible to apply the proposed approach for effectively mapping monopole sources in wind tunnel tests.
\end{abstract}

Keywords: Localization, parameter estimation, acoustic imaging, sparsity constraint, robust super-resolution

\section{Introduction}

Acoustic imaging is widely used for acoustic source power reconstruction and localization. It can provide the useful insights into acoustic performance, acoustic comfort and machinery security in automobile and aeronautic industries for wind tunnel tests [1-4]. In this article, we mainly focus on the signal processing techniques applied in acoustic imaging, such as the Conventional BeamForming $(\mathrm{CBF})$, deconvolution and regularization methods. The CBF method [5] is a direct, robust and rough estimation of source powers and positions, since its spatial resolution is limited due to the high side-lobes. The MUltiple SIgnal

\footnotetext{
Article partly based on that accepted at the IEEE International Symposium on Signal Processing and Information Technology (ISSPIT2011) pp 286-289, Bilbao, Spain, Dec.14-17,2011.

*Corresponding author: Ning.CHU@lss.supelec.fr. Tel. : +33 (0)1 6985 1743. Fax : 0033 (0)1 69851765

${ }^{1}$ The author's PhD study is financed by China Scholarship Council (CSC) and École Supérieure d'Électricité (SUPELEC) France

Classification (MUSIC) [6] can greatly improve the CBF resolution, but original MUSIC requires the high signal-tonoise ration (SNR) or the exact number of sources to make the subspace separation. Besides, the MUSIC could not directly reconstruct source powers due to its pseudo-power optimization. Based on the CBF, the acoustic power propagation can be modeled by a determined linear system of equations, which could hardly be solved by direct inversions due to the invertible propagation matrix. Therefore, the deconvolution methods, like the CLEAN [7], can iteratively extract strong sources from the blurry beamforming powers. But the CLEAN could leave out weak sources interfered by strong background noises; and some important parameters of CLEAN have to be empirically selected for good performance. Recently, the Deconvolution Approach for Mapping of Acoustic Source (DAMAS) [8] has become a breakthrough and been effectively applied in acoustic imaging for wind tunnel tests by the NASA. The DAMAS 
can iteratively solve the acoustic power propagation model under the non-negative constraint on source power variables. But the dominant drawback of the DAMAS is the sensitivity to background noises. So that the Diagonal Removal (DR)-DAMAS [8] has been proposed for the noise suppression; however, weak sources could be also removed off by the DR-DAMAS. To overcome the deconvolution drawbacks, the DAMAS with sparsity constraint (SC-DAMAS) [9] can greatly improve the spatial resolution and improve the robustness, but SC-DAMAS could cause overweening effects due to the sparsity parameter selection. The Covariance Matrix Fitting (CMF) method [10] can effectively improve the robustness by jointly estimating the source power covariance matrix and background noise power; however, the original CMF is not feasible to use because of its huge dimensionality of variables in covariance matrix. For robust acoustic imaging, the Spectral Estimation Method (SEM) and its extensions $[11,12]$ are proposed to subtract the reference noise power from the measured data; and this reference noise power can be obtained beforehand by measuring the observed signals without any object in wind tunnel. However, the estimated noise power might be different from the case where the object is installed in the wind tunnel. Furthermore, sparse regularization methods [13-15] have been widely developed by using the $\ell_{1}$-norm. However, some of them have to carefully select the regularization parameter, or make necessary approximations on Singular Value Decomposition (SVD). More recently, the Bayesian inference approaches [16-19] have been investigated and achieve more robust and better acoustic imaging results. However, the Bayesian framework often causes very timeconsuming computation costs for real applications.

To summarize, all the above state-of-the-art methods have excellent performance on their own applications, but there is no one-fits-all methods; and most of them suffer one of the following drawbacks: coarse spatial resolution, sensitivity to background noises and high computational cost. In addition, most of them need to set some important parameters for good performance.

In this article, our main contributions can be: 1) We firstly improve the robust forward propagation model of acoustic power propagation by considering both the background noises at the microphone sensors, and the propagation uncertainty caused by multi-path propagation in the wind tunnel. 2) We jointly estimate source powers and positions, as well as the background noise power. 3) For acoustic imaging with super-resolution, we investigate an adaptive sparsity parameter estimation procedure. 4) Furthermore, its computational cost maintains feasible to use.

This article is organized as follows: Section 2 introduces the forward model of acoustic signal propagation. Then the improved model of acoustic power propagation is proposed in Section 3. The classical methods are presented in Section 4. Our proposed approach is investigated in Section 5. Then method comparisons are shown on simulations in Section 6 and real data in Section 7. To further confirm the effectiveness of proposed approach, Section 8 demonstrates the performance comparisons on the hybrid data, in which, some known synthetic sources are added to the real data. Finally, Section 9 concludes this article.

\section{Forward model of acoustic signal propagation}

\subsection{Assumptions}

For acoustic imaging, a source is usually supposed to be an uncorrelated monopole $[7-9,11,20-22]$. In this article, we use the monopole model in order to simplify the physical process and explicitly build up the acoustic propagation model. To approach real cases, we use the complex source model which is composed of several monopoles forming different spatial patterns. Moreover, we suppose the background noise at the microphone sensor to be Additive Gaussian White Noise (AGWN), mutually independent and identically distributed (i.i.d), and 


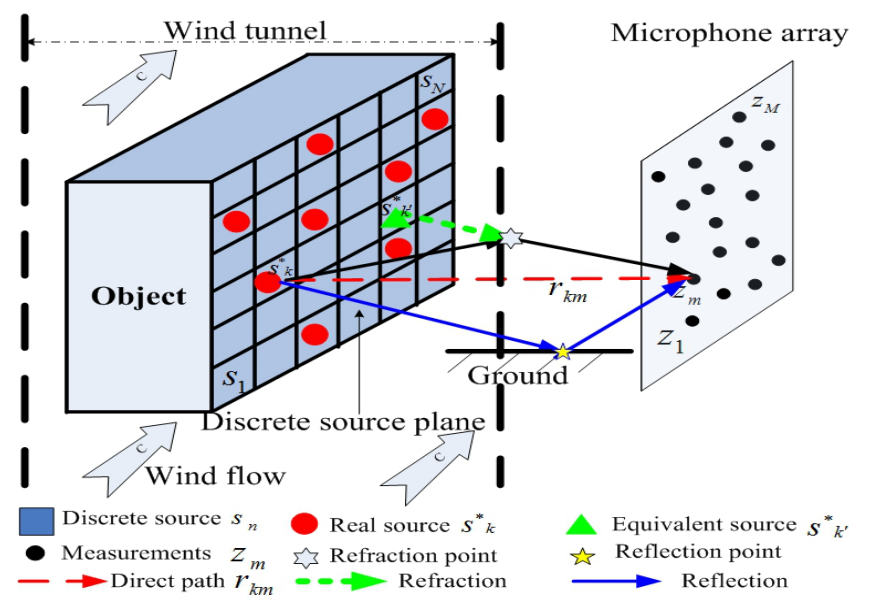

Figure 1: Illustration of acoustic propagation in the wind tunnel [19].

also independent to sources. Sensors are assumed to be omni-directional with unitary gain. Furthermore, complex reverberations are negligible in wind tunnel, but we consider the first order reflection on the ground, as well as the refraction on the interface between the wind flow and common air.

\subsection{Acoustic signal propagation}

Figure 1 illustrates the acoustic signal propagation from the source plane to the microphone sensor array in the wind tunnel, where sensors are installed outside the wind flow. We consider $M$ sensors at known positions $\overline{\mathbf{P}}=$ $\left[\overline{\mathbf{p}}_{1}, \cdots, \overline{\mathbf{p}}_{M}\right]^{T}$ with $(\cdot)^{T}$ denotes transpose operator. On the source plane, we suppose $K$ unknown original source signals $\mathbf{s}^{*}=\left[s_{1}^{*}, \cdots, s_{K}^{*}\right]^{T}$ at unknown positions $\mathbf{P}^{*}=$ $\left[\mathbf{p}_{1}^{*}, \cdots, \mathbf{p}_{K}^{*}\right]^{T}$, where $\mathbf{p}_{k}^{*}$ denotes the $3 \mathrm{D}$ coordinates of $s_{k}^{*}$. Then we discretize the source plane into $N$ identical grids at known discrete positions $\mathbf{P}=\left[\mathbf{p}_{1}, \cdots, \mathbf{p}_{N}\right]^{T}$, where we assume that $K$ original sources sparsely distribute on these grids, supposing $\mathrm{N}>\mathrm{M}>>\mathrm{K}$ and $\mathbf{P}^{*} \subset \mathbf{P}$. Finally we get $N$ discrete source signals $\mathbf{s}$ at known positions $\mathbf{P}$ as:

$$
\mathbf{s}=\left[0, \cdots, s_{1}^{*}, 0, \cdots, s_{k}^{*}, 0, \cdots, s_{K}^{*}, 0, \cdots\right]_{N}^{T}
$$

where $s_{k}^{*}=s_{n}$ for $\mathbf{p}_{k}^{*}=\mathbf{p}_{n}$. Since $K<<N$, thus $\mathbf{s}$ is a sparse signal with K-sparsity in the spatial domain. Therefore, to reconstruct original source signals $\mathbf{s}^{*}$ is transferred to reconstruct K-sparsity signals $\mathbf{s}$. To be clear, we state that $\mathbf{s}^{*}=\left[s_{1}^{*}, \cdots, s_{K}^{*}\right]^{T}$ denote the original source signals, while $\mathbf{s}=\left[s_{1}, \cdots, s_{N}\right]^{T}$ denotes the (discrete) source signals. In Eq.(1), source position $\mathbf{p}_{k}^{*}$ can be derived from the position $\mathbf{p}_{n}$, where the source power of $s_{n}$ is not 0 .

Based on the discrete source model in Eq.(1), we can give the forward model of acoustic signal propagation. For the $m$ th sensor $m \in[1, \cdots, M]$, received signals $\mathbf{z}_{i, m}(t)$ are divided into $I$ sampling blocks with $L$ samplings/block, with sampling block $i \in[1, \cdots, I]$, sampling time $t \in$ $[(i-1) L+1, \cdots, i L]$ and total samplings $T=I L$. Since acoustic signals usually have wide-band frequencies, we apply the L-points Discrete Fourier Transform (DFT) in each sampling block, so that we separate the wide-band into $L$ narrow frequency bins. Since the signal processing is made independently at each frequency bin, we omit the frequency notation $f_{l}, l \in[1, \cdots, L]$ for simplicity. Finally in the sampling block $i$, the measured signals $\mathbf{z}_{i}=$ $\left[z_{i, 1}, \cdots, z_{i, M}\right]^{T}$ at $M$ senors can be modeled in the frequency domain as [20]:

$$
\mathbf{z}_{i}=\mathbf{A}(\mathbf{P}) \mathbf{s}_{i}+\mathbf{e}_{i}
$$

where $\mathbf{s}_{i}=\left[s_{i, 1}, \cdots, s_{i, N}\right]^{T}$ denotes $\mathrm{N}$ source signals at the $i$ th sampling block. After DFT, $\mathbf{s}_{i}$ still maintains the sparsity in spatial domain; and $\mathbf{e}_{i}=\left[e_{i, 1}, \cdots, e_{i, M}\right]^{T}$ denotes background noises at $\mathrm{M}$ sensors, and we suppose $\mathbf{e}_{i} \sim \mathcal{N}\left(0, \sigma^{2}\right)$ to be the i.i.d AGWN distribution, where $\sigma^{2}=\mathbb{E}\left[\mathbf{e}_{i}^{H} \mathbf{e}_{i}\right]$ denotes the noise power, with $\mathbb{E}[\cdot]$ denoting expectation operator and $(\cdot)^{H}$ conjugate transpose. $M \times N$ complex matrix $\mathbf{A}(\mathbf{P})=\left[\mathbf{a}\left(\mathbf{p}_{1}\right), \cdots, \mathbf{a}\left(\mathbf{p}_{N}\right)\right]$ denotes the signal propagation matrix, where $\mathbf{a}\left(\mathbf{p}_{n}\right)$ denotes the steering vector for the source $s_{n}$ at the position $\mathbf{p}_{n}$. As shown in Fig.(1), we can modify the classical definition [20] of $\mathbf{a}\left(\mathbf{p}_{n}\right)$ according to the ground reflection on the ground as follows:

$$
\mathbf{a}_{n}=\mathbf{a}_{d}\left(\mathbf{p}_{n}\right)+\rho \mathbf{a}_{r}\left(\mathbf{p}_{-n}\right),
$$

where $\rho$ denotes the reflection coefficient $(0 \leq \rho \leq 1)$, whose value mainly depends on ground conditions at a 
given frequency. For the real data used in this article, $\rho=0.8$ is supposed to be fixed over the frequency band $[1600,2600] \mathrm{Hz}$ in the wind tunnel experiments, thanks to the research contributions of Renault SAS [23].

$$
\mathbf{a}_{d}\left(\mathbf{p}_{n}\right)=\left[\frac{1}{r_{n, 1}} e^{-\mathrm{\jmath} 2 \pi f_{l} \tau_{n, 1}}, \cdots, \frac{1}{r_{n, M}} e^{-\mathrm{\jmath} 2 \pi f_{l} \tau_{n, M}}\right]^{T} \text { is }
$$

the direct steering vector, where $r_{n, m}=\sqrt{\left\|\mathbf{p}_{n}-\overline{\mathbf{p}}_{m}\right\|^{2}}$ denotes the propagation distance from source $n$ to sensor $m$; and $\tau_{n, m}$ denotes the propagation time within $r_{n, m}$; if the media is uniform, $r_{n, m}$ is thus the geometry distance and $\tau_{n, m}=r_{n, m} / c_{0}$ with $c_{0}$ being the acoustic speed.

$\mathbf{a}_{r}\left(\mathbf{p}_{-n}\right)=\left[\frac{1}{r_{-n, 1}} e^{-\mathrm{\jmath} 2 \pi f_{l} \tau_{-n, 1}}, \cdots, \frac{1}{r_{-n, M}} e^{-\mathrm{\jmath} 2 \pi f_{l} \tau_{-n, M}}\right]^{T}$ denotes the reflect steering vector, where $\mathbf{p}_{-n}$ denotes the symmetric position of $\mathbf{p}_{n}$ according to the ground. For $r_{n, m}$ and $\tau_{n, m}$ on the real data in Section 7 , we can apply mirror sources to correct the ground reflection. Moreover, since it is not an uniform media from source plane to sensors in the wind tunnel as shown in Fig.1, we apply the equivalent sources to correct the wind refraction as discussed in authors' article [19]. Without corrections, the imaging results of real data will have position shifts due to the wind refraction, as well as ghost shadows due to the ground reflection.

In short, the forward model of signal propagation in Eq.(2) is a linear system of equations for discrete source signals $\mathbf{s}$, since the measured signals $\mathbf{z}$ are known and signal propagation matrix $\mathbf{A}(\mathbf{P})$ can be calculated from Eq.(3) based on source plane discretization. However, Eq.(2) is under-determined, since the number of equations $M$ is less than the number $N$ of unknown signals.

\section{Proposed forward model of power propagation}

As we have stated in the Introduction, acoustic imaging mainly involves the source power reconstruction and localization. According to Eq.(2), we can estimate source signals $\mathbf{s}$ from measured signals $\mathbf{z}$, then we can calculate the acoustic powers $\mathbf{x}=\left[x_{1}, \cdots, x_{N}\right]^{T}$ of uncorrelated sources by $\mathbf{x}=\operatorname{diag}\left[\mathbf{R}_{s}\right]$ with $\mathbf{R}_{s}=\mathbb{E}\left[\mathbf{s s}^{H}\right]$ being the source covariance matrix and diag[.] being diagonal items. However, to solve the Eq.(2) confront two difficulties: 1). Multisolutions due to under-determined equations; 2). Source signals s are complex, which contain both amplitude and phase variables.

In order to overcome these obstacles and enhance the solution robustness, we directly build up the forward model of acoustic power propagation by considering the background noises at the sensor array, as well as the propagation uncertainty caused by multi-path propagation in the wind tunnel. Based on the measured signals $\mathbf{z}_{i}$ in Eq.(2), a rough estimation of source powers can be directly obtained by the CBF method [4] as:

$$
y_{n}=\mathbb{E}\left[\tilde{\mathbf{a}}_{n}^{H} \mathbf{z}_{i}\right]^{2}=\tilde{\mathbf{a}}_{n}^{H} \mathbf{R} \tilde{\mathbf{a}}_{n}
$$

where $y_{n}$ denotes the beamforming power at the position $\mathbf{p}_{n}$ on the source plane; and $y_{n}$ can be an estimated source power for $x_{n}$; and $\tilde{\mathbf{a}}$ denotes the beamforming steering (back-projection) vector at the position $\mathbf{p}_{n}$, defined as

$$
\tilde{\mathbf{a}}_{n}=\frac{\mathbf{a}_{n}}{\left\|\mathbf{a}_{n}\right\|_{2}^{2}},
$$

where $\mathbf{a}_{n}$ is the signal propagation steering vector defined in Eq.(3); $\|\cdot\|_{2}$ denotes the vector $\ell_{2}$-norm; $\mathbf{R}$ denotes the measured covariance matrix, defined as:

$$
\mathbf{R}=\mathbb{E}\left[\mathbf{z}_{i} \mathbf{z}_{i}^{H}\right]=\sum_{n=1}^{N} x_{n} \mathbf{a}_{n} \mathbf{a}_{n}^{H}+\sigma^{2} \mathbf{I}_{M},
$$

where $x_{n} \in \mathbf{x}$ denotes the source power of $s_{n}$ at position $\mathbf{p}_{n}$; and $\mathbf{I}_{M}$ denotes the $\mathrm{M} \times \mathrm{M}$ identity matrix. In practice, $\mathbf{R}$ is estimated by $\hat{\mathbf{R}}$, defined as

$$
\hat{\mathbf{R}}=\frac{1}{I} \sum_{i=1}^{I} \mathbf{z}_{i} \mathbf{z}_{i}^{H}
$$

where $\mathbf{z}_{i}$ denotes measured signals at the $i$ th sampling block in Eq.(2); $I$ is the total number of sampling blocks. If the sampling block number $I>>1$, we have $\hat{\mathbf{R}} \approx \mathbf{R}$ in Eq.(7). Therefore, replacing $\mathbf{R}$ in Eq.(4) by $\hat{\mathbf{R}}$, we then obtain the forward model of acoustic power propagation in the vector form as follows:

$$
\mathbf{y}=\mathbf{C} \mathbf{x}+\sigma^{2} 1_{a}+\boldsymbol{\xi}
$$


where $\mathbf{y}=\left[y_{1}, \cdots, y_{N}\right]^{T}$ is the beamforming vector obtained from Eq.(4). So that Eq.(8) becomes the determined linear system of equations for source powers $\mathbf{x}$, compared with the under-determined forward model of signal propagation in Eq.(2). $1_{a}=\left[\frac{1}{\left\|\mathbf{a}_{1}\right\|^{2}}, \cdots, \frac{1}{\left\|\mathbf{a}_{N}\right\|^{2}}\right]^{T}$ is a constant vector.

$$
\boldsymbol{\xi}=\left[\xi_{1}, \cdots, \xi_{N}\right]^{T} \text { denotes the propagation uncertainty, }
$$
which represents the remaining unknown effects due to the wind reflection and refraction happened at other positions rather than the ground or wind tunnel boundary.

$$
\mathbf{C}=\left[c_{n, q}\right] \text { with } n, q \in[1, \cdots, N] \text { denotes power propa- }
$$
gation matrix [8], whose item $c_{n, q}$ is defined [19] as:

$$
c_{n, q}=\left(\tilde{\mathbf{a}}_{n}^{H} \mathbf{a}_{q}\right)^{2}
$$

where beamforming steering vector $\tilde{\mathbf{a}}_{n}$ and signal propagation steering vector $\mathbf{a}_{n}$ are defined in Eq.(5) and Eq.(3) respectively. In fact, $c_{n, q}$ represents the power contribution of the $q$ th source to the $n$th position on the source plane. If the microphone array is ideal enough, $c_{n, q}$ becomes the Dirac function as $c_{n, q}=\delta_{n, q}$ with $\delta_{n, q}=1$ for $n=q$; and $\delta_{n, q}=0$ for $n \neq q$. Then Eq.(8) becomes $\mathbf{y}=\mathbf{x}+\sigma^{2} 1_{a}+\boldsymbol{\xi}$, which reveals that the beamforming powers $\mathbf{y}$ measured at the sensors is composed of source powers $\mathbf{x}$, background noise power $\sigma^{2}$ and the unknown powers due to propagation uncertainty.

In brief, according to the improved forward model of acoustic power propagation in Eq.(8), $\mathbf{x}$ can be estimated from $\mathbf{y}$ by minimizing propagation uncertainty $\boldsymbol{\xi}$.

\section{Classical deconvolution and sparsity methods}

In Eq.(8), unfortunately, $\mathbf{C}$ is usually a singular matrix [8] and cannot be invertible. But according to the definition of $\mathbf{C}$ in Eq.(9), equation (8) can be interpreted as a kind of deconvolution problem. One of the recently developed deconvolution methods is the DAMAS [8], which assumes $\sigma_{e}^{2}=0$ and $\boldsymbol{\xi}=0$ in Eq.(8) and tries to iteratively solve $x_{n}^{(i+1)}=y_{n}-\sum_{q=1}^{n-1} c_{n, q} x_{q}^{(i+1)}-\sum_{q=n+1}^{N} c_{n, q} x_{q}^{(i)}$ with $x_{n} \geq 0$, where $(i)$ denotes the $i$ th iteration. However, sensitivity to the noise could be the main drawback of the DAMAS. Then the DR-DAMAS is proposed by setting $\operatorname{diag}[\hat{\mathbf{R}}]=0$ in Eq.(7) so as to suppress the noises, but DR technique inevitably harms weak sources whose powers are lower than the noises.

To well solve Eq.(8), sparse regularization methods [9, 10, 13-15, 24] have been widely applied as follows:

$$
\left\{\begin{array}{l}
\hat{\mathbf{x}}=\arg \min _{(\mathbf{x})}\left\{\|\mathbf{y}-\mathbf{C x}\|_{2}^{2}+\alpha\|\mathbf{x}\|_{1}\right\} \\
\text { s.t. } \quad \mathbf{x} \succeq 0
\end{array}\right.
$$

where the first $\ell_{2}$-norm $\|\cdot\|_{2}$ represents the data fitting part; the second $\ell_{1}$-norm $\|\cdot\|_{1}$ enforces the sparsity solution of $\mathbf{x}$, and greatly improves the spatial resolutions; $\ell_{2}+\ell_{1}$ optimization has been well solved by the LASSO [25] and atomic decomposition via basis pursuit [22, 26]; the third term $\alpha$ denotes regularization parameter, which has to be tuned carefully [26-28] for good performance.

The sparse regularization in Eq.(10) is equivalent to the sparsity constraint as:

$$
\left\{\begin{array}{l}
\hat{\mathbf{x}}=\arg \min _{(\mathbf{x})}\left\{\|\mathbf{y}-\mathbf{C x}\|_{2}^{2}\right\} \\
\text { s.t. } \quad\|\mathbf{x}\|_{1}=\beta, \quad \mathbf{x} \succeq 0
\end{array}\right.
$$

where $\beta$ denotes the total source power; $\|\mathbf{x}\|_{1}=\beta$ serves the sparsity constraint; $\mathbf{x} \succeq 0$ denotes $x_{n} \in \mathbf{x} \geq 0$. Recently, many effective methods have been proposed to solve Eq.(11), such as the DAMAS with sparsity constraint (SCDAMAS) [9]. But $\beta$ selection is the key issue for good performance. Similarly using the sparsity constraint, the Covariance Matrix Fitting (CMF) method [10] can directly estimate the signal covariance matrix as:

$$
\left\{\begin{array}{l}
\left(\hat{\mathbf{x}}, \sigma^{2}\right)=\arg \min _{\left(\mathbf{x}, \sigma^{2}\right)}\left\{\left\|\hat{\mathbf{R}}-\mathbf{A} \mathbf{X} \mathbf{A}^{H}-\sigma^{2} \mathbf{I}_{M}\right\|_{2}^{2}\right\} \\
\text { s.t. } \operatorname{tr}[\mathbf{X}]=\beta, \mathbf{x} \succeq 0, \sigma^{2} \geq 0
\end{array},\right.
$$

where $\hat{\mathbf{R}}$ is the measured covariance matrix in Eq.(7); $\mathbf{X}=\mathbb{E}\left[\mathbf{s s}^{H}\right]$ denotes the source power covariance matrix; $\operatorname{tr}[\cdot]$ denotes the matrix trace. The CMF can estimate the correlated sources, but it has much larger dimension of variables to be estimated than the methods in Eq.(10-11). 


\section{Proposed approach using sparsity constraint}

Compared with classical sparse regularization method in Eq.(10-12), we want to jointly estimate the source powers $\mathbf{x}$ and background noise power $\sigma^{2}$ by minimizing the propagation uncertainty $\boldsymbol{\xi}$. In order to obtain super resolution in strong background noises, we adaptively estimate the sparsity parameter $\beta$ on total source power. Therefore, our proposed robust super-resolution approach with sparsity constraint (SC-RDAMAS) is expressed as:

$$
\left\{\begin{array}{l}
\left(\hat{\mathbf{x}}, \hat{\sigma^{2}}\right)=\arg \min _{\left(\mathbf{x}, \sigma^{2}\right)}\left\{\left\|\mathbf{y}-\mathbf{C x}-\sigma^{2} 1_{a}\right\|_{2}^{2}\right\} \\
\text { s.t. } \quad \mathbf{x} \succeq 0, \quad\|\mathbf{x}\|_{1}=\beta, \quad \sigma^{2} \geq 0
\end{array}\right.
$$

where sparsity parameter $\beta$ is the total power of source signals, so that $\beta$ is defined as:

$$
\beta=\sum_{k=1}^{K} x_{k}^{*}=\left\|\mathbf{x}^{*}\right\|_{1}=\sum_{n=1}^{N} x_{n}=\|\mathbf{x}\|_{1}=\operatorname{tr}[\mathbf{X}],
$$

where $\mathrm{K}$ is the total number of original source signals $\mathbf{s}^{*}$; and $\mathbf{x}^{*}=\operatorname{diag}\left[\mathbb{E}\left[\mathbf{s}^{*} \mathbf{s}^{* H}\right]\right]$ denotes the original source powers; $\mathbf{x}=\operatorname{diag}\left[\mathbb{E}\left[\mathbf{s s}^{H}\right]\right]$ denotes the (discrete) source powers; $\mathbf{X}=\mathbb{E}\left[\mathbf{s s}^{H}\right]$ denotes the source power covariance matrix. If $\beta$ in Eq.(14) is modeled too large, the estimated $\hat{\mathbf{x}}$ from Eq.(13) would be more dispersed than expected; if $\beta$ too small, some of weak sources would be left out. Therefore, the adaptive estimation of sparsity parameter $\beta$ is an essential issue in the proposed approach.

\subsection{Adaptive estimation of sparsity parameter}

According to the definition of measured covariance matrix $\mathbf{R}$ in Eq.(6), we take the matrix trace as:

$$
\begin{aligned}
\operatorname{tr}[\mathbf{R}] & =\operatorname{tr}\left[\mathbf{A} \mathbf{X} \mathbf{A}^{H}\right]+M \sigma^{2} \\
& =\sum_{n=1}^{N}\left\|\mathbf{a}_{n}\right\|^{2} x_{n}+M \sigma^{2}
\end{aligned},
$$

where $\mathbf{a}_{n}$ is the $n$th column of signal propagation matrix $\mathbf{A}$, defined in Eq.(3). Let $\|\mathbf{a}\|_{\min }$ and $\|\mathbf{a}\|_{\max }$ respectively denote the minimum and maximum vector norms within $\mathbf{a}_{n}, n=[1, \cdots, N]$. From Eq.(15), we have

$\frac{1}{\|\mathbf{a}\|_{\max }^{2}}\left(\operatorname{tr}[\mathbf{R}]-M \sigma^{2}\right) \leq\|\mathbf{x}\|_{1} \leq \frac{1}{\|\mathbf{a}\|_{\min }^{2}}\left(\operatorname{tr}[\mathbf{R}]-M \sigma^{2}\right)$ where $\|\mathbf{x}\|_{1}=\sum_{n=1}^{N} x_{n}$ and $x_{n} \geq 0$ in Eq.(14). Since $\mathbf{R}$ is also a Hermitian matrix, it can be diagonalized as $\operatorname{tr}[\mathbf{R}]=$ $\operatorname{tr}\left[\mathbf{U} \boldsymbol{\Lambda} \mathbf{U}^{H}\right]=\operatorname{tr}[\boldsymbol{\Lambda}]$, where $\mathbf{U}$ is the unitary matrix, whose columns are eigenvectors of $\mathbf{R}$; and $\boldsymbol{\Lambda}$ is the eigenvalue matrix of $\mathbf{R}$. According to Eq.(14-16), we can obtain $\beta$ estimation as:

$$
\hat{\beta}=\frac{1}{2}\left(\frac{1}{\|\mathbf{a}\|_{\max }^{2}}+\frac{1}{\|\mathbf{a}\|_{\min }^{2}}\right)\left(\operatorname{tr}[\hat{\mathbf{\Lambda}}]-M \hat{\sigma}^{2}\right),
$$

where $\hat{\boldsymbol{\Lambda}}$ is the eigenvalue matrix of $\hat{\mathbf{R}}$ in Eq.(7); and $\hat{\sigma^{2}}$ can be estimated as [10]:

$$
\hat{\sigma^{2}}=\frac{1}{M-\hat{K}} \sum_{m=\hat{K}+1}^{M} \hat{\lambda}_{m}
$$

where $\hat{\lambda}_{m}$ denotes the eigenvalue of $\hat{\mathbf{R}}$, satisfying $\hat{\lambda}_{1} \geq$ $\cdots \geq \hat{\lambda}_{K} \geq \hat{\lambda}_{K+1}=\cdots=\hat{\lambda}_{M}=\hat{\sigma}^{2}$; and $M$ is the total sensor number; $\hat{K}$ denotes the estimated source number, provided $\hat{K} \in[1, \cdots, M]$. When $\hat{K}=M$, we have noise power $\hat{\sigma}^{2}=0$.

In Eq.(17), source number $K$ estimation plays an importance role in determining $\beta$. There are many methods for source number estimation such the SVD[13] and Bayesian framework [29]. Our paper [30] gives a fast and rough estimation on $K$ as: let $\mathcal{F}\left(\lambda_{m}\right)=\mathcal{F}\left[\lambda_{1}, \cdots, \lambda_{M}\right]$ with $m \in[1, \cdots, M]$ denote the eigenvalue distribution function, where $\mathcal{F}[\cdot]$ denotes the interpolation. The first $K$ big eigenvalues should contain the $K$ original source powers, while the latter $M-K$ eigenvalues just equal the noise power $\sigma^{2}$. So that $\mathcal{F}\left(\lambda_{m}\right)$ has a sparse distribution. Suppose $\mathcal{F}\left(\lambda_{m}\right)$ to be second derivative, when its curvature $\kappa(\hat{K}) \approx 0$ within $\hat{K} \in[1, \cdots, M]$, we can thus get $K \approx \hat{K}$. However, since the under-estimation of source number could eliminate the weak sources, it is better to initialize $K$ as a relative big value. Therefore, we can use the upper bound of source number as discussed in compressed sensing [31-33] as:

$$
K=\|\mathbf{x}\|_{0} \leq \frac{1}{2}\left(1+\frac{1}{\mu}\right)
$$


Algorithm 1 Proposed adaptive estimation procedure

1. Input:

Signal propagation matrix A in Eq.(2);

measured signal covariance matrix $\hat{\mathbf{R}}$ in Eq.(7);

measured beamforming powers $\mathbf{y}$ in Eq.(4);

power propagation matrix $\mathbf{C}$ in Eq.(9);

\section{Initialization:}

Iteration number $\mathrm{i}=1$;

source number $\hat{K}^{(1)}=\frac{1}{2}\left(1+\frac{1}{\mu}\right)$ in Eq.(19);

variables $\hat{\mathbf{x}}^{(1)}=0 ;{\hat{\sigma^{2}}}^{(1)}=0$;

criterion $\mathcal{J}\left(\hat{\mathbf{x}}^{(1)}, \hat{\sigma}^{2}{ }^{(1)}\right)=0, \mathcal{J}\left(\hat{\mathbf{x}}^{(2)},{\hat{\sigma^{2}}}^{(2)}\right)=1$;

\section{Iterations:}

While $\left|\mathcal{J}\left(\hat{\mathbf{x}}^{(i+1)},{\hat{\sigma^{2}}}^{(i+1)}\right)-\mathcal{J}\left(\hat{\mathbf{x}}^{(i)},{\hat{\sigma^{2}}}^{(i)}\right)\right|$ is not small enough; Else: Steps 4;

3.1 Update: $\hat{\sigma}^{(i)}$ by Eq.(18), $\hat{\beta}^{(i)}$ by Eq.(17);

\subsection{Optimize:}

Solve Eq.(13) by interior point algorithm [34]:

$\left(\hat{\mathbf{x}}^{(i+1)},{\hat{\sigma^{2}}}^{(i+1)}\right)=\arg \min _{\left(\mathbf{x}, \sigma^{2}\right)}\left\{\mathcal{J}\left(\hat{\mathbf{x}}^{(i)},{\hat{\sigma^{2}}}^{(i)}\right)\right\}$

s.t. $\|\mathbf{x}\|_{1} \leq \hat{\beta}^{(i)}, \hat{\mathbf{x}}^{(i+1)} \geq 0,{\hat{\sigma^{2}}}^{(i+1)} \geq 0$;

3.3 Iterate: $\hat{K}^{(i+1)}=\hat{K}^{(i)}-1, \mathrm{i}=\mathrm{i}+1$;

4. Output: $\hat{\mathbf{x}}, \hat{\sigma^{2}}, \hat{K}, \hat{\beta}$, then Stop.

where $\mu=\max _{(1 \leq i \neq j \leq M)} \frac{\hat{\mathbf{R}}_{i}^{H} \hat{\mathbf{R}}_{j}}{\left\|\hat{\mathbf{R}}_{i}\right\|\left\|\hat{\mathbf{R}}_{j}\right\|}$ denotes the incoherence of the measured covariance matrix $\hat{\mathbf{R}}$, where $\hat{\mathbf{R}}_{i}$ denotes its $i$ th column vector. According to Eq.(6) and independence assumption between sources and noises, $\mu$ can reflect the incoherence of source power covariance matrix $\mathbf{X}$.

\subsection{Proposed adaptive estimation procedure}

In Eq.(13), our proposed approach is a convex quadratic minimization under linear matrix constraints, which can be solved by interior point algorithms using MATLAB toolbox SeMuDi [34]. In order to improve the robustness to background noises $\sigma^{2}$ and sparsity parameter $\beta$, we propose an adaptive estimating algorithm as depicted in Algorithm 1: Let $\mathcal{J}\left(\mathbf{x}, \sigma^{2}\right)=\left\|\mathbf{y}-\mathbf{C x}-\sigma^{2} 1_{a}\right\|_{2}^{2}$ define the cost function ; we firstly initialize source number $\mathrm{K}$ by using the matrix incoherence $\mu$ of Eq.(19); then $\hat{\sigma}^{2}$ is obtained from Eq.(18) and $\hat{\beta}$ from (17); and then we simultaneously estimate source powers $\mathbf{x}$ and $\sigma^{2}$ by using the interior point algorithm [34]; finally we update $\hat{K}^{(i+1)}=\hat{K}^{(i)}-1$ for a new estimation.

\subsection{Power estimation of wide-band acoustic signals}

In wind tunnel tests, acoustic sources are usually generated by wind frictions against the car surface. Different car parts produce different characteristic frequencies. Therefore, acoustic signals have the wide frequency band. In Section 2, we have taken DFT transformation and separated the wide-band into $L$ independent frequency bins, then we have engaged signal processing in each frequency bin. Using the proposed SC-RDAMAS approach in Eq.(13), we can obtain $\hat{\mathbf{x}}\left(f_{l}\right)$ as the estimation of source power $\mathbf{x}\left(f_{l}\right)$ at $l$ th frequency bin. Finally, total power $\mathbf{x}_{w b}$ over the wide-band $\left[f_{\min }, f_{\max }\right]$ can be estimated by averaging the summation of estimated result in each frequency bin as: $\hat{\mathbf{x}}_{w b}=\frac{1}{L} \sum_{f_{l}=f_{\min }}^{f_{\max }} \hat{\mathbf{x}}\left(f_{l}\right)$.

\section{Simulations on uncorrelated source imaging}

This section shows the typical simulations on source power reconstruction and localization of monopole sources. The proposed SC-RDAMAS approach is compared with 5 classical methods in the poor SNR cases. Reconstruction results are presented via images which can directly show the estimated source powers $(\mathrm{dB})$ and positions. Then we use 3 criteria to quantitatively evaluate estimation performance. The first one is the averaged estimation error of original source powers, defined as $\overline{\Delta x^{*}}=\frac{1}{K} \sum_{k=1}^{K}\left|\hat{x}_{k}^{*}-x_{k}^{*}\right|$, where original source powers $\mathbf{x}^{*}=\left[x_{1}^{*}, \cdots, x_{K}^{*}\right]^{T}$ are defined as $\mathbf{x}^{*}=\operatorname{diag}\left[\mathbb{E}\left[\mathbf{s}^{*} \mathbf{s}^{* H}\right]\right]$. The second is the reconstruction error of source power image, defined as $\delta_{2}=$ $\frac{\|\hat{\mathbf{x}}-\mathbf{x}\|_{2}^{2}}{\|\mathbf{x}\|_{2}^{2}}$. Compared with $\overline{\Delta x^{*}}$, the value of $\delta_{i}$ not only depends on the estimated positions and powers, but also on the suppression of background noise power. The last is the averaged position error, defined as $\overline{\Delta p^{*}}=\frac{\sqrt{\sum_{k=1}^{K}\left(\left\|\mathbf{p}_{k}^{*}-\hat{\mathbf{p}}_{k}^{*}\right\|\right)}}{\sqrt{\sum_{k=1}^{K}\left\|\mathbf{p}_{k}^{*}\right\|}}$, 
where $\hat{\mathbf{p}}_{k}^{*}$ denotes the estimated positions of $k$ th original source.

In Fig.1, simulation configurations are almost the same as the wind tunnel experiments carried out by Renault SAS [35]. For example, $D=4.50 \mathrm{~m}$ is the distance between the sensor plane and source plane. There are $M=64$ non-uniform sensors locating on the vertical plane. This Non-Uniform sensor Array (NUA) has a longer horizontal size than the vertical [35]. And it has the $d=2 \mathrm{~m}$ averaged size. The advantage of NUA array is that it can yield almost the same performance but less computation burden than the uniform array with the same sensors as discussed in Ref.[36]. $c_{0} \approx 340 \mathrm{~m} / \mathrm{s}$ is the acoustic speed in the common air. $T=10000$ is the total number of samplings, which is large enough to meet the important condition for the bearforming in Eq.(4) and improved forward model of power propagation in Eq.(8). To focus on method comparisons, we do not consider the ground reflection and wind refraction in simulations. But we should reconsider the multi-path propagation effects in wind tunnel experiments in Section 7.

For the simulated sources in Fig.2(a), we have simulated 4 monopoles and 5 complex sources with different patterns. And these source are spaced at least $20 \mathrm{~cm}$. We take $K \in[9,23]$ as the value range of the total source number. Original source powers $\mathbf{x}^{*}$ are within $[0.08,2]([-$ $10.3,3.7] \mathrm{dB}$ ) and $14 \mathrm{~dB}$ dynamic range. For the image result on Fig.2(a), there are 4 parts: the center image shows the source positions, patterns and powers; on its right, the colormap shows the dynamic range of source powers, in which, the dark-red colors represent strong powers, while light white colors represent the weak; on the left and bottom, two profile figures reveal the positions of 4 monopoles and complex monopole source on the center. To simulate the very noisy background, the noise power is set $\sigma^{2}=0.86$ $(-0.7 \mathrm{~dB})$, thus the averaged SNR is $0 \mathrm{~dB}$.

In order to make a fair comparison with other classical methods, some simulation parameters should be selected
Table 1: Power estimations of 4 monopole sources by average power estimation error $\overline{\Delta x^{*}}$, relative errors of power image reconstruction $\delta_{2}$ and estimated noise power $\hat{\sigma}^{2}$ at $2500 \mathrm{~Hz}, \mathrm{SNR}=0 \mathrm{~dB}$, simulated $\sigma^{2}=0.86$; '-' means unavailable.

\begin{tabular}{|l|c|c|c|c|c|c|c|}
\hline Source power & 0.08 & 0.18 & 0.98 & 0.50 & $\overline{\Delta x^{*}}$ & $\delta_{2}$ & $\hat{\sigma}^{2}$ \\
\hline CBF & 1.57 & 11.28 & 3.51 & 2.02 & 69.64 & 121.9 & - \\
\hline DAMAS & - & - & - & 0.44 & 3.14 & 1.33 & - \\
\hline CLEAN & - & 0.25 & 0.44 & 0.28 & 0.87 & 0.67 & - \\
\hline SC-DAMAS & - & - & - & - & 1.03 & 0.58 & - \\
\hline DR-DAMAS & - & - & 0.77 & 0.23 & 0.30 & 0.08 & - \\
\hline CMF & 0.09 & - & 0.80 & 0.40 & 0.31 & 0.10 & 0.89 \\
\hline Proposed & 0.09 & 0.10 & 1.05 & 0.43 & 0.06 & 0.06 & 0.85 \\
\hline
\end{tabular}

carefully. In order to avoid the spatial aliasing problem as discussed in the DAMAS [8], the discrete grid is set $\Delta p=5 \mathrm{~cm}$ and the frequency should be $f<3100 \mathrm{~Hz}$, so that they satisfy $\frac{\Delta p}{\delta_{p}^{C B F}}<0.2$, where $\delta_{p}^{C B F}=\frac{D c_{0}}{d f}$ denotes the spatial resolution of the CBF. To simulate a sparse distribution of discrete source signals, $\Delta p=5 \mathrm{~cm}$ is used to discretize the $100 \times 150 \mathrm{~cm}^{2}$ source plane, so that the power image is of $21 \times 31$ pixels. Since total grid number $N=651$ is much more larger than original source number $K=23$, the discrete source signals $\mathbf{s}$ and their source powers $\mathbf{x}$ are both K-sparsity signals.

\subsection{Method comparisons}

Firstly we show the method comparisons at $2500 \mathrm{~Hz}$, since this frequency is very sensitive to human hearing and affect acoustic comfort. In Fig.2, the CBF gives an obscure image of source power distributions; the DAMAS with 5000 iterations (5000i), CLEAN and SC-DAMAS well detect some of strong sources, but they do not provide reliable estimation of weak sources in strong background noises; the DR-DAMAS effectively removes the noise interference, but some of weak sources are also removed off; the CMF achieves better estimation on the noise power and distinguishes most of sources; however, it fails to reconstruct some patterns of weak sources.

In Fig.2(h), proposed SC-RDAMAS approach not only detects most of the complex sources, but also well recon- 


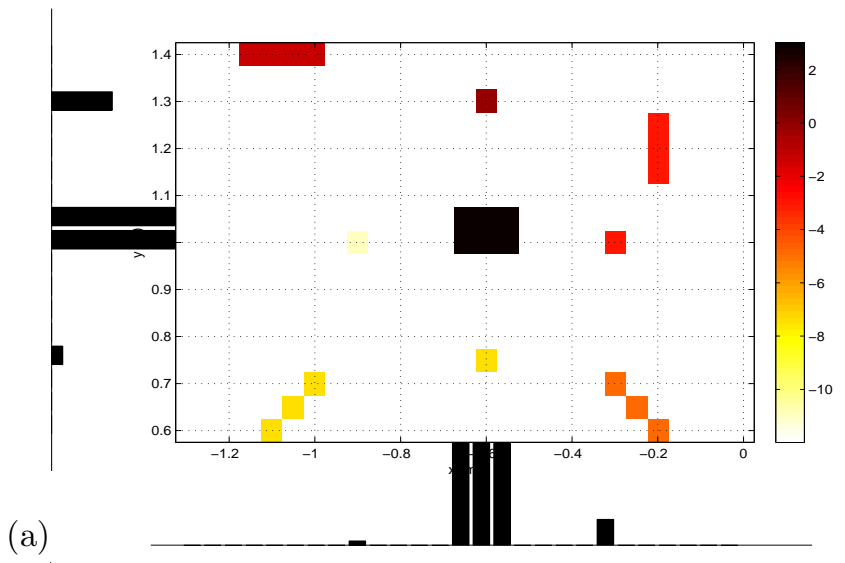

(b)
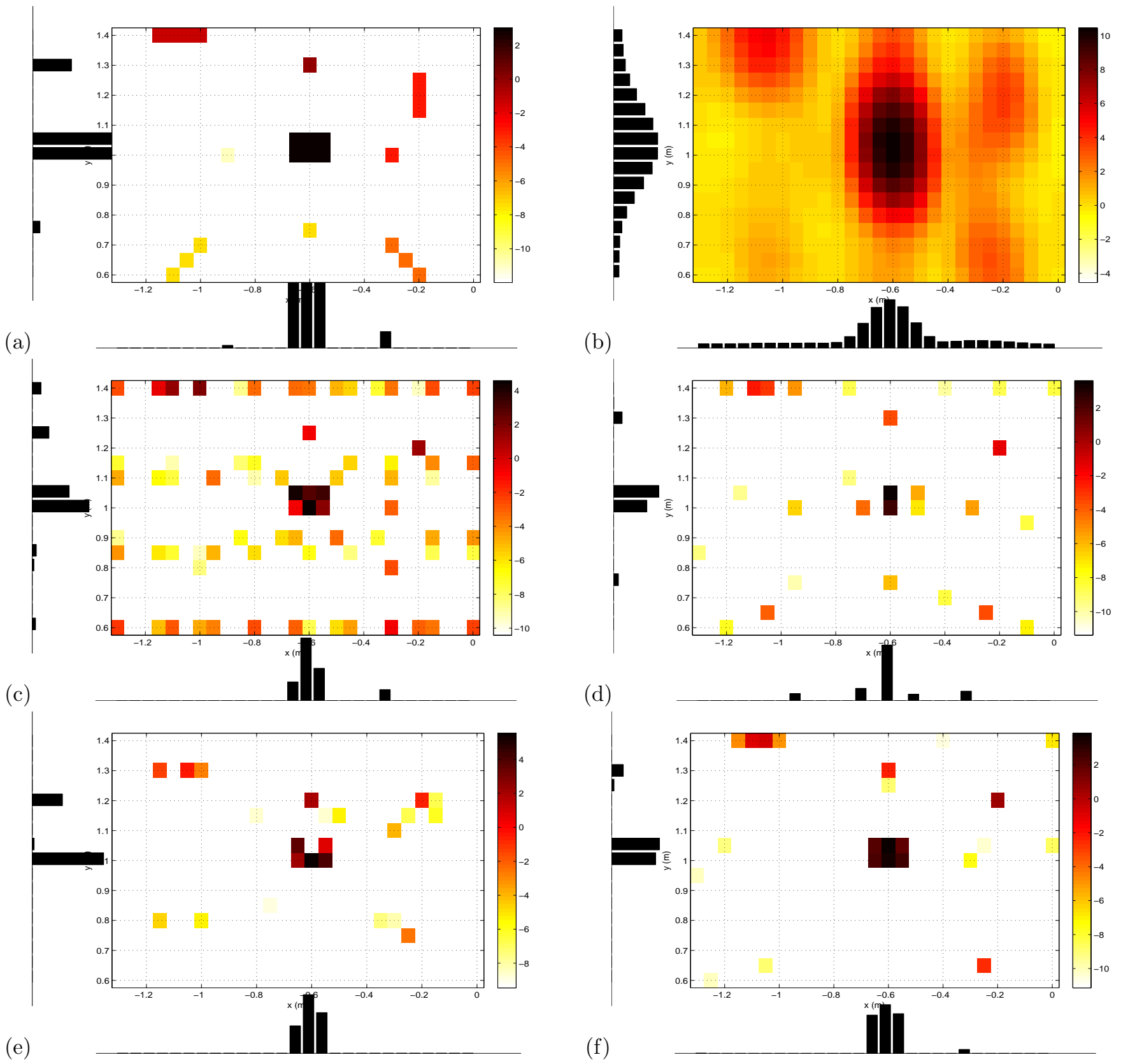

(d)

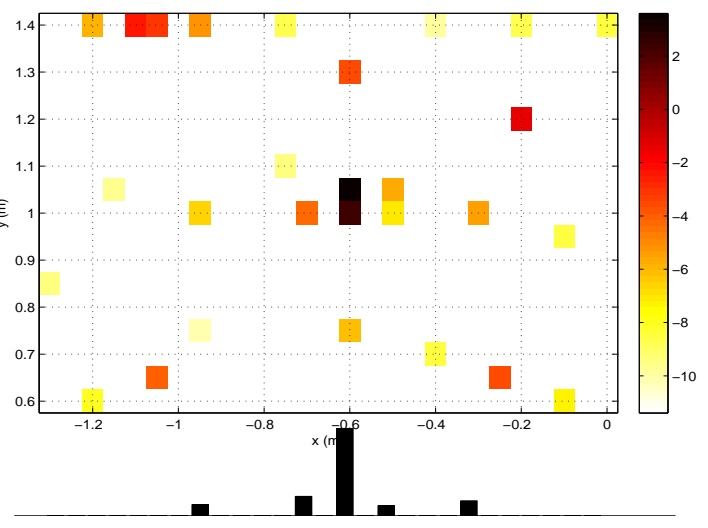

(e)

(f)
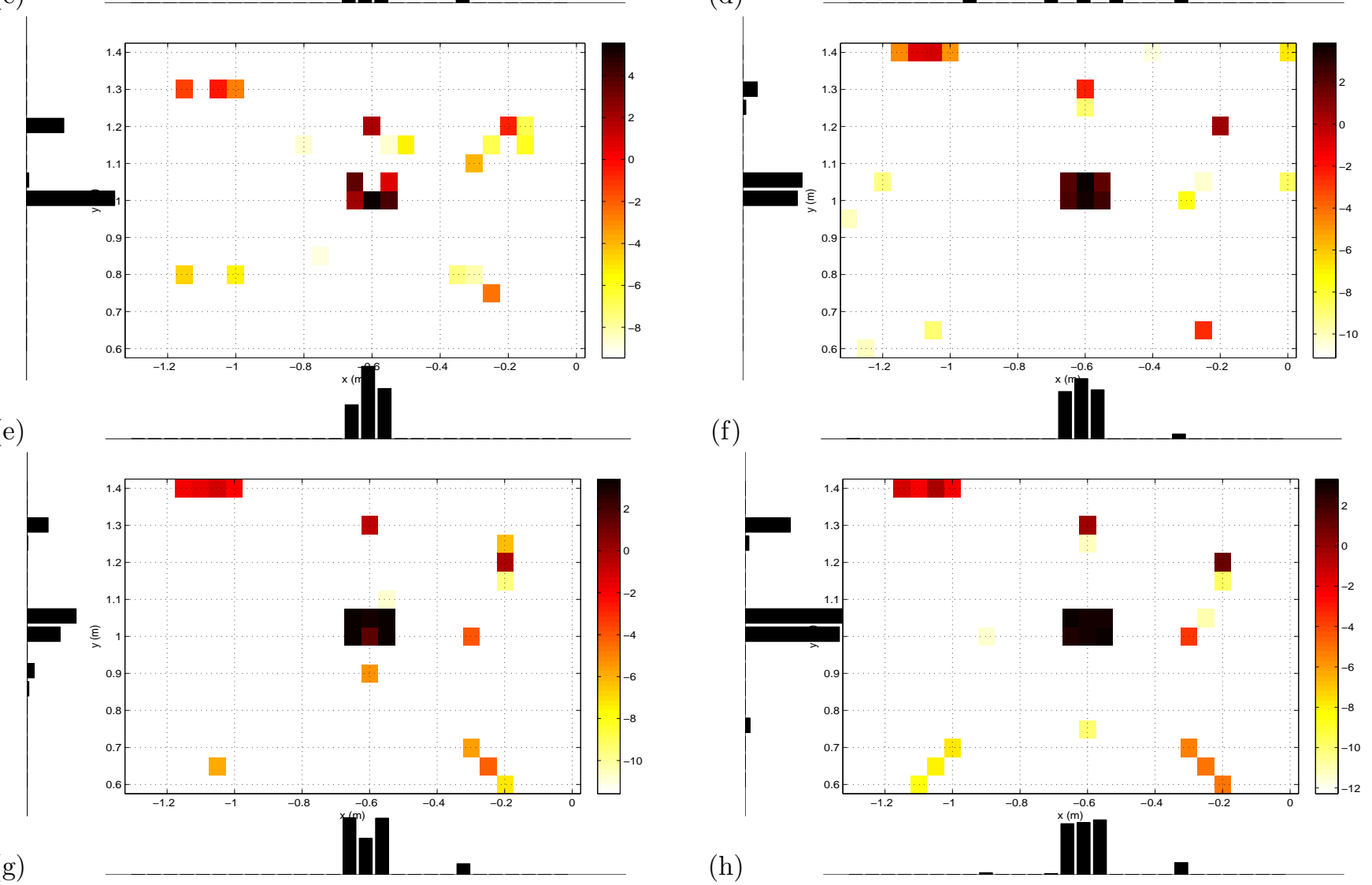

(h)

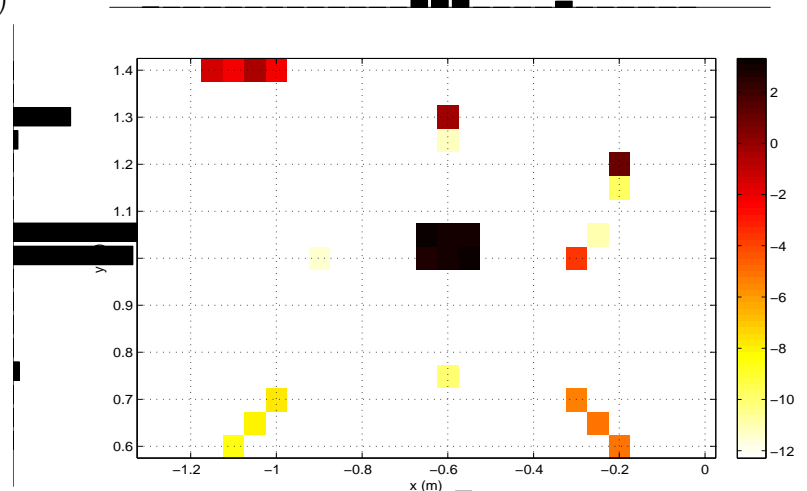

Figure 2: Simulation on monopole sources with $14 \mathrm{~dB}$ power dynamic range at $2500 \mathrm{~Hz}, \sigma^{2}=0.86, \mathrm{SNR}=0 \mathrm{~dB}$ and $15 \mathrm{~dB}$ display: (a) Monopole sources (b) CBF (c) DAMAS with 5000 iterations (5000i) (d) CLEAN (e) SC-DAMAS (f) DR-DAMAS (5000i) (g) CMF and (h) Proposed SC-RDAMAS 
Table 2: Position estimations of 4 monopole sources by averaged position errors $\overline{\Delta p^{*}}$ at $2500 \mathrm{~Hz}, \mathrm{SNR}=0 \mathrm{~dB}$; '-' means unavailable.

\begin{tabular}{|l|c|c|c|c|c|}
\hline Position & $(-0.9,1)$ & $(-0.6,0.75)$ & $(-0.3,1)$ & $(-0.6,1.3)$ & $\overline{\Delta p^{*}}$ \\
\hline CBF & - & - & - & - & 1 \\
\hline DAMAS & - & $(-0.6,0.85)$ & $(-0.3,1)$ & $(-0.6,1.25)$ & 0.28 \\
\hline CLEAN & $(-0.95,1)$ & $(-0.6,0.75)$ & $(-0.3,1)$ & $(-0.6,1.3)$ & 0.01 \\
\hline SC-DAMAS & - & - & - & $(-0.6,1.2)$ & 0.63 \\
\hline DR-DAMAS & - & - & $(-0.3,1)$ & $(-0.6,1.3)$ & 0.43 \\
\hline CMF & - & $(-0.6,0.9)$ & $(-0.3,1)$ & $(-0.6,1.3)$ & 0.29 \\
\hline Proposed & $(-0.9,1)$ & $(-0.6,0.75)$ & $(-0.3,1)$ & $(-0.6,1.3)$ & 0 \\
\hline
\end{tabular}

Table 3: Power estimations of the complex monopole source on the center of image by power estimation error $\overline{\Delta x^{*}}$ at $2500 \mathrm{~Hz}$, $\mathrm{SNR}=0 \mathrm{~dB}$; '-' means unavailable.

\begin{tabular}{|l|c|c|c|c|c|c|c|}
\hline Source power & 2.00 & 2.00 & 2.00 & 2.00 & 2.00 & 2.00 & $\overline{\Delta x^{*}}$ \\
\hline CBF & 2.64 & 9.60 & 9.70 & 9.64 & 11.34 & 9.77 & 6.78 \\
\hline DAMAS & 4.50 & 1.25 & 0.48 & 2.54 & 0.49 & 1.88 & 1.15 \\
\hline CLEAN & 2.29 & 0.37 & 1.69 & - & 0.27 & 0.34 & 1.27 \\
\hline SC-DAMAS & 1.68 & 2.49 & 1.16 & 0.10 & 2.23 & 0.65 & 0.75 \\
\hline CMF & 1.36 & 2.86 & 2.07 & 2.09 & 1.92 & 1.05 & 0.45 \\
\hline DR-DAMAS & 2.15 & 2.05 & 1.82 & 1.83 & 2.50 & 1.45 & 0.27 \\
\hline Proposed & 1.83 & 2.00 & 2.05 & 1.72 & 2.16 & 1.95 & 0.12 \\
\hline
\end{tabular}

structs source powers and positions in poor SNR situation. According to the adaptive estimation procedure in Algorithm $1, \hat{K}=25$ is better initialized owing to $\mu \approx 0.02$ from Eq.(19). According to the three quantitative criteria $\overline{\Delta x^{*}}, \overline{\Delta p^{*}}$ and $\delta_{2}$ in the Table 1,2 and 3 respectively, the proposed approach works much better than the others: 1) Compared with the low spatial resolutions of the $\mathrm{CBF}$ $\left(\delta_{p}^{C B F}=31 \mathrm{~cm}\right.$ at $\left.2500 \mathrm{~Hz}\right)$, proposed approach makes good use of sparse distribution of source powers and it achieves the resolution as high as $5 \mathrm{~cm}$ in both horizontal and vertical directions. Since the horizontal aperture of NUA array is larger than the vertical, all the results of classical methods obtain better horizontal resolution. But our proposed approach still achieves the super resolution in vertical direction. 2) Compared with the sensitiveness of deconvolution methods, proposed approach greatly improves the robustness by jointly estimating the background noise as well as the source powers. 3) Compared with sparse reg-

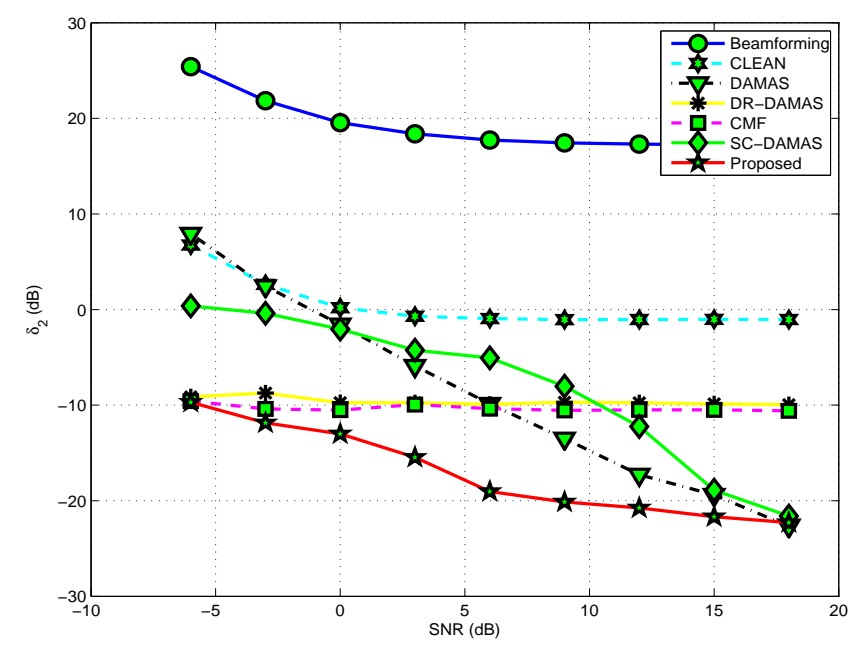

Figure 3: Performance comparison for relative errors of power image reconstruction $\delta_{2}$ versus $\mathrm{SNR}[-6,18] \mathrm{dB}$ on simulations at $2500 \mathrm{~Hz}$.

ularization methods, we adaptively estimate the sparsity parameter on the total source power, so that fits well for the strong i.i.d AGWN noise. To make a fair comparison, we realize the CMF, SC-DAMAS and proposed SCRDAMAS based on Matlab toolbox SeMuDi [34].

In Fig.3, we show the relative error of power image reconstruction $\delta_{2}$ of mentioned methods within SNR [-6, $18 \mathrm{~dB}$ at $2500 \mathrm{~Hz}$. Proposed approach is more robust to background noises than other classical methods.

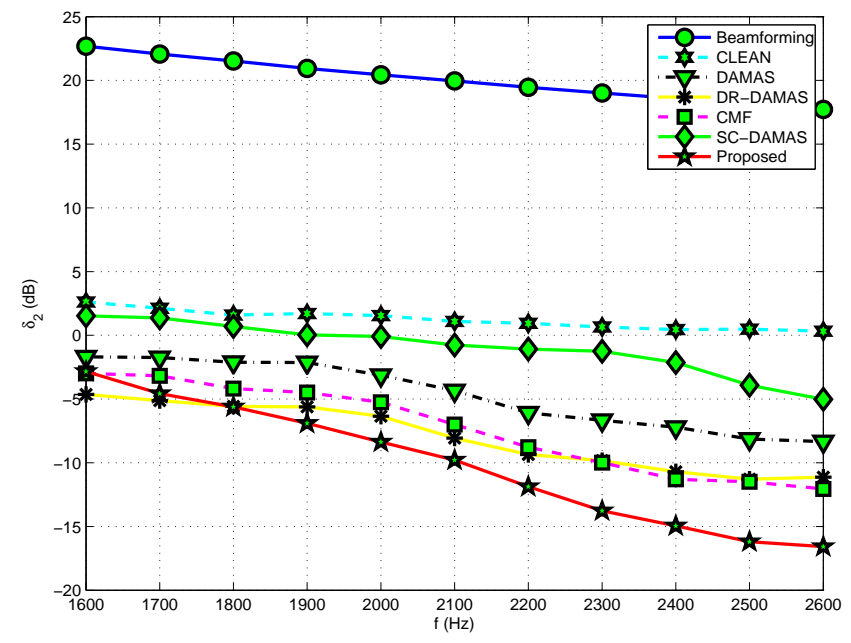

Figure 4: Performance comparison for relative errors of power image reconstruction $\delta_{2}$ versus $[1600,2600] \mathrm{Hz}$ on simulations at $\mathrm{SNR}=3 \mathrm{~dB}$.

In Fig.4, we show reconstruction errors $\delta_{2}$ versus different frequency bins within $[1600,2600] \mathrm{Hz}$ which affects 
the acoustic comfort of human being. The SNR is set $3 \mathrm{~dB}$. At high frequencies, proposed approach provides the most significant improvements. At low frequencies, proposed approach still maintains small reconstruction errors.

\subsection{Overweening effects of proposed approach}

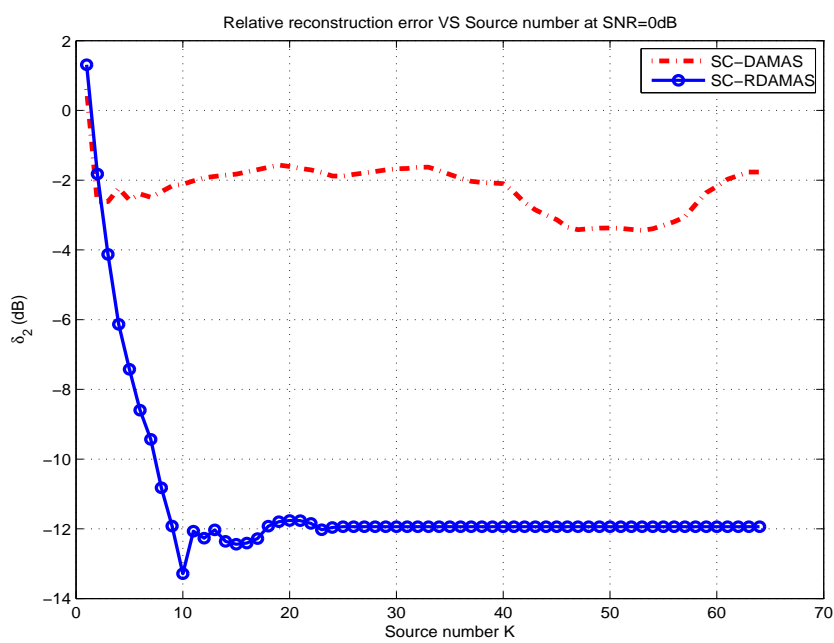

Figure 5: Sparsity parameter influence caused by estimated source number $\hat{K}$ versus power image reconstruction error $\delta_{2}$ between the SC-DAMAS and proposed SC-RDAMAS at 0dB and $2500 \mathrm{~Hz}$.

One of the common limitations in the SC-DAMAS, CMF and proposed SC-RDAMAS, is the overweening effect which is well known in the compressed sensing[3133]: the sparse results are often composed of discontinuous and unstructured (shapeless) points rather than continuous source distributions. Though these shapeless points could represent the monopole source power to some extent, they could hardly present distributed sources. Taking Fig.2(e)(g) for example, the SC-DAMAS and CMF can only obtain discontinuous monopoles for complex sources. In Fig.2(h), though proposed SC-RDAMAS well detects most of the sources, it could barely discover one complex source whose continuous pattern is a vertical line. Moreover, the artifacts on the three figures are always monopole points which are near to sources.

The first reason for the above overweening effects is the sparsity parameter influence. In Fig.5, we compare the sparsity parameter influence on $\delta_{2}$ error between the SCDAMAS and proposed SC-RDAMAS at 0dB and $2500 \mathrm{~Hz}$. The sparsity constraint is interpreted by the total source power $\beta$ so as to regularize data fitting errors in Eq.(11) and Eq.(13) respectively. And $\beta$ in Eq.(17) mainly depends on the source number $K$ estimation, when background noise is supposed to be i.i.d AGWN. Therefore, to evaluate the sparsity parameter is equivalent to evaluate the source number estimation. Since complex sources in Fig.2(a) are supposed to be made of uncorrelated monopoles, it is reasonable to take $K \in[9,23]$. In Fig. 5 , when $\hat{K}<9$ is under-estimated, it is clear to see that $\delta_{2}$ error of the two methods are both very sensitive to $\hat{K}$, so that neither of them could obtain a good reconstruction. When $9 \leq \hat{K} \leq 23$, proposed approach can obtain smaller $\delta_{2}$ errors, but both of the two methods could hardly achieve stable results. When $\hat{K}>23$ is over-estimated, proposed SC-RDAMAS steadily keeps much smaller $\delta_{2}$ than the SCDAMAS. Above all, proposed SC-RDAMAS can well initialize source number $K$ and adaptively estimate sparsity parameter $\beta$ compared with the SC-DAMAS.

Secondly, the sparsity constraint on total source power could not appropriately model the source sparse distributions nor source structures. Suppose two different source power distributions: $\mathbf{x}_{1}=[1,2,3,0,0,0,0,0,0]^{T}$ and $\mathbf{x}_{2}=$ $[3,2,1,0,0,0,0,0,0]$ which have the same total source power $\beta=6$. To reconstruct $\mathbf{x}_{1}$ and $\mathbf{x}_{2}$ from their beamforming data $\mathbf{y}_{1}$ and $\mathbf{y}_{2}$ respectively in the case of very strong background noises, it is highly necessary to investigate other sparsity prior models $[19,33,37]$ instead of only using sparsity constraint $\beta=6$. Therefore, in Fig.2(h), our approach can hardly detect all the complex sources with different sparse distributions.

The last but not least, the monopole assumption on acoustic model is too simple to model the complex sources with different patterns. Therefore, in Fig.2(h), our approach tends to generate unexpected monopole artifacts near to sources, especially when the SNR is as small as 


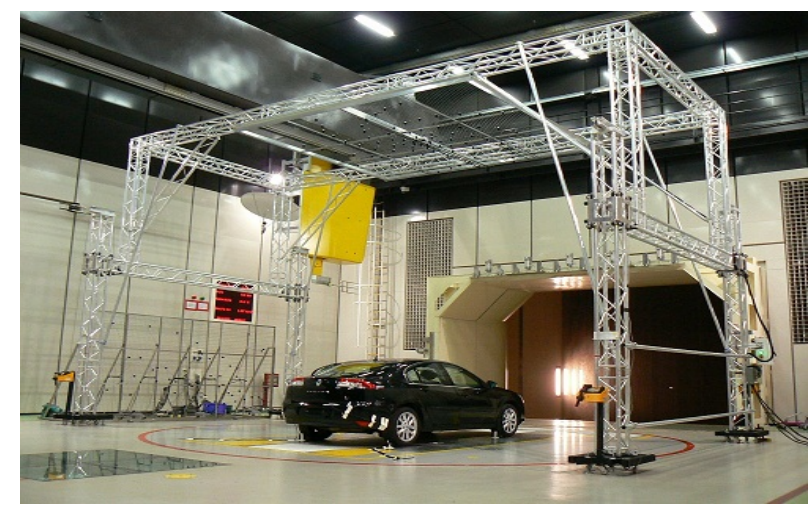

Figure 6: Wind tunnel S2A [35] in France.

0dB. For the real data in Section 7, this drawback will become the dominant reason, and more obvious overweening effects will be seen.

\section{Wind tunnel experiments}

Figure 6 shows the static vehicle (no engine noise), microphone sensor array and the wind flow at the speed of $160 \mathrm{~km} / \mathrm{h}$ in the wind tunnel S2A [35]. One of objects of this wind tunnel is to detect acoustic powers and positions on the car surface. This wind tunnel can simulate a traveling car on the high-way and measure its acoustic comfort to the passengers-by.

\subsection{Experiment configurations}

We suppose that all acoustic sources locate on the same 2D plane, since the curvature of the car side is relatively small compared with the distance $\mathrm{D}=4.5 \mathrm{~m}$ between the car and array plane. The surface of car side is of $150 \times 500$ $\mathrm{cm}^{2}$, and we discretize this source plane into $31 \times 101$ pixels by using identical grid $\Delta p=5 \mathrm{~cm}$; and we also focus on a small region of the rear-view mirror: $1 \times 1.5 \mathrm{~m}^{2}(21 \times 31$ pixels). In the real data, there are $\mathrm{T}=524288$ samplings with the sampling frequency $f_{s}=2.56 \times 10^{4} \mathrm{~Hz}$. As discussed in Section 2, we separate these samplings into $I=204$ blocks with $L=2560$ samplings per block. The working frequency band is chosen as $[2400,2600] \mathrm{Hz}$, which is sensitive to acoustic comfort of human being. The image results are shown by normalized dB images with 10dB span. For the acoustic imaging on the vehicle surface in wind tunnel tests in Fig.(6), acoustic sources often sparsely locate on the rear-view mirrors and around the wheels, while on the rest parts, there are few significant sources. Therefore, the discrete source signals $\mathbf{s}$ and their powers $\mathbf{x}$ are both sparse signals. But unfortunately, we do not know the exact source number or SNR beforehand. Therefore, it is necessary to investigate the adaptive estimation procedure in Algorithm 1 for robust acoustic imaging in wind tunnel tests. Moreover, we consider the ground reflection in Eq.(3) and wind refraction [19] in both synthetic and real data.

\subsection{Results of single frequency at $2500 \mathrm{~Hz}$}

Figure.7 illustrates the estimated power images of mentioned methods at $2500 \mathrm{~Hz}$. In Fig.7(a), the CBF merely gives a blurred image of strong sources around the front wheel, rear-view mirror and back wheel. In Fig.7(b), the DAMAS well deconvolves the beamforming image, and discovers weak sources on the front light, front cover and side window; however, many false targets are also detected in the air. In Fig.7(c), DR-DAMAS eliminates most of the artifacts, but it also removes off some of weak sources. Figure.7(d) shows that the CLEAN overcomes the drawbacks of the DAMAS, but we have to carefully select the parameters for this good performance. In Fig.7(e), the SCDAMAS has a better noise suppression than the DAMAS and CLEAN owing to the sparsity parameter selection, but SC-DAMAS overwhelms too much both the noises and the sources, so that it does not provide a wide dynamic range of source power estimations.

Finally in Fig.7(f), proposed SC-RDAMAS not only manages to distinguish the strong sources around the two wheels, rear-view mirror and side window, but also successfully reconstructs the week ones on the front cover and light. In fact, the proposed adaptive estimation procedure in Algorithm 1 inevitably increases more computational 
(a)

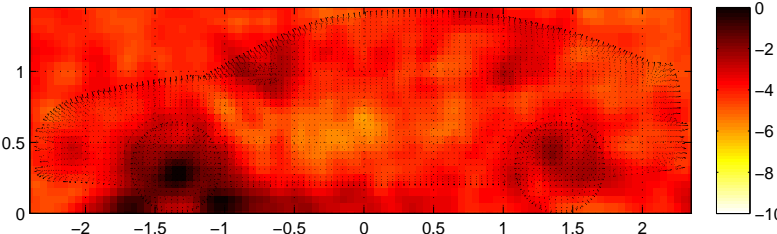

(c)

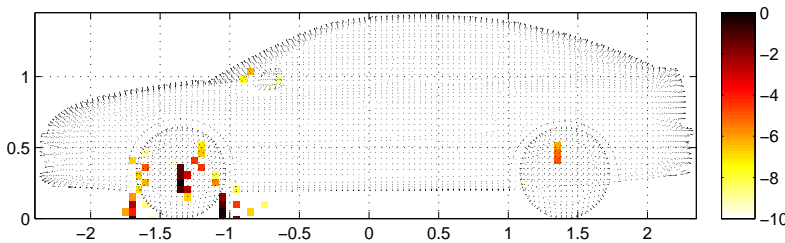

(e)

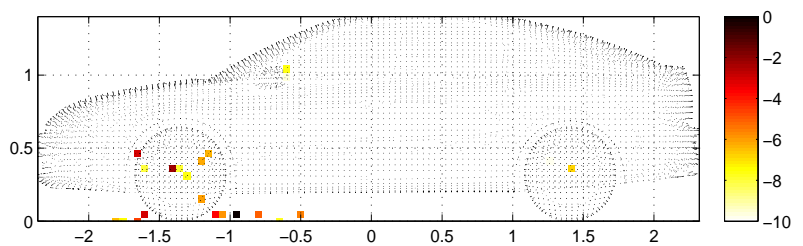

(b)

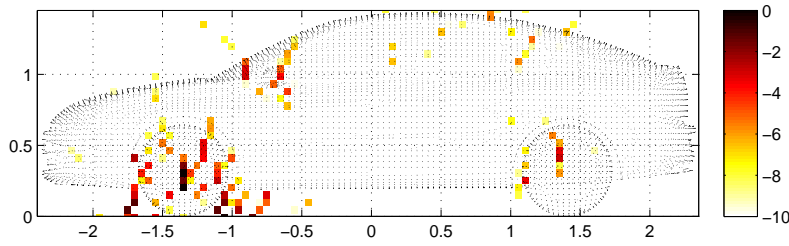

(d)
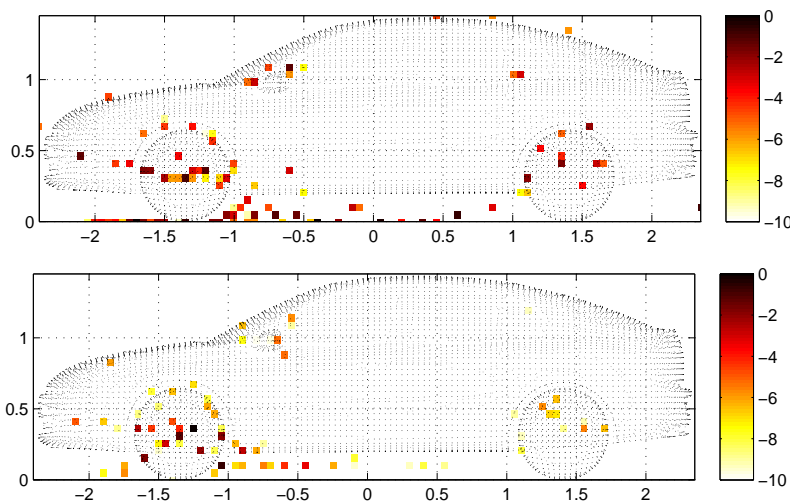

Figure 7: Acoustic imaging of real data on the whole car side at 2500Hz: (a) CBF (b) DAMAS (5000i) (c) DR-DAMAS (5000i) (d) CLEAN (e) SC-DAMAS and (f) Proposed SC-RDAMAS.

Table 4: Computational cost for treating whole car: image $30 \times 100$ pixels, at $2500 \mathrm{~Hz}$, based on CPU:3.33GHz, '-' means unavailable.

\begin{tabular}{|c|c|c|c|c|c|c|c|}
\hline Methods & CBF & DAMAS (5000i) & DR-DAMAS (5000i) & CLEAN & Proposed & SC-DAMAS & CMF \\
\hline Time (s) & 1 & 10 & 11 & 45 & 852 & 1254 & Very Long \\
\hline
\end{tabular}

cost than the deconvolution methods such as DAMAS and CLEAN. But our approach still remains a moderate complexity compared with sparse regularization methods such as the SC-DAMAS as shown in Table 4. Due to the high dimension of variables in source power covariance matrix, we cannot realize the original CMF method on real data.

Based on the acoustic imaging on the car side, we investigate a small part of the rear-view mirror. In Fig.8(a), the CBF detects strong sources on the corner of the front wheel and rear-view mirror. The DAMAS in Fig.8(b) improves the spatial resolutions, but it causes some unexpected spots. In Fig.8(c), the DR-DAMAS eliminates most of false spots. In Fig.8(e)-(f), the CMF, SC-DAMAS and proposed approach achieve much better resolutions and offer more details of source power distributions on the rear-view mirror. (a)

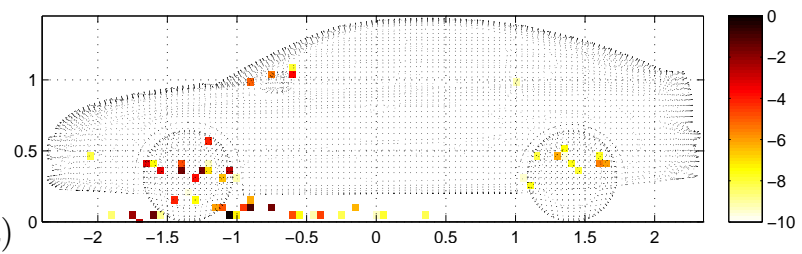

(b)

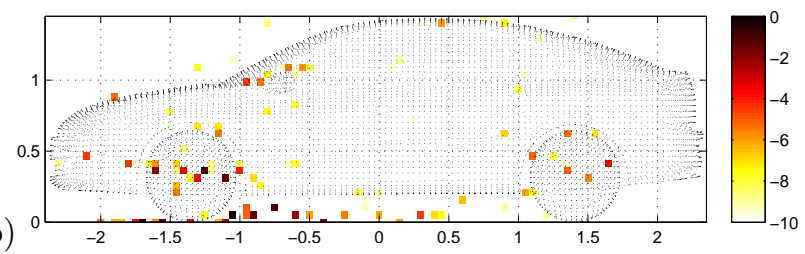

Figure 9: Sparsity parameter influence of proposed SC-RDAMAS on real data at $2500 \mathrm{~Hz}$ : (a) Under-estimated $\hat{\beta}$ (b) Over-estimated $\hat{\beta}$.

\subsection{Overweening effects of proposed approach}

The overweening effects caused by the sparsity constraint in Fig.7(e)(f) are more obvious than the simulations in Fig.2(e)(g)(h). This phenomenon on the real data could be explained by the following facts:

Source model problem. Some of acoustic sources on the car surface (wheels and rearview mirrors) might be 
distributed sources which have structures and patterns. Thus the monopole source model used in this article could not fit any more. Since the sparsity constraint $\left(\ell_{1}\right.$ norm) on total source power enforces the monopole reconstructions and neglects source structures to some extent, the mentioned sparsity methods prefer to offer the discontinuous point results, as typically shown on the back wheel in Fig.7(e)(f).

Sparsity parameter problem. In proposed approach, the estimated sparsity parameter $\hat{\beta}$ in Eq.(17) depends on the source number and background noise power. On real data, however, it is hard to estimate source number $K$ on the car surface. Moreover, background noises in the wind tunnel are not always i.i.d AGWN noises. Consequently, it is not easy to exactly derive $\hat{\beta}$ from Eq.(17). In Fig.9(a), if $\hat{\beta}$ is under-estimated, some weak sources would be inevitably lost on the headlight, top antenna and side window; and source patterns on the rearview mirror and back wheel would be roughly expressed by several discontinuous points. If $\hat{\beta}$ is over-estimated, both weak sources and source patterns could be better detected, but the artifacts would be produced in the air and under the car body. Similarly overweening effects can also be seen in Fig.8(d)(f) and Fig.10(c) and (d). But according to the adaptive sparsity parameter estimation procedure in Algorithm 1, our proposed approach try to avoid under-estimate $\beta$.

\subsection{Results of wide-band data}

Based on the imaging results at single frequency, we show performance comparisons of wide-band data within [2400, 2600] Hz which affects the acoustic comfort. In Fig.10, each method obtains a clearer result than the correspondent one at $2500 \mathrm{~Hz}$ in Fig.7. This is because that source powers are enforced, but flashing false targets are suppressed over the wide-band average. The reconstruction of DAMAS in Fig.10(a) is reasonable, but its spatial resolution is not high enough on the front wheel and rearview mirror. Figure.10(b) shows that the CLEAN greatly ameliorates the resolution, but unexpected points under the car caused by the ground reflection should be further eliminated; the SC-DAMAS in Fig.10(c) has the advantages of the CLEAN, but it could not detect the weak sources around the back wheel due to the sparsity parameter selection. Finally in Fig.10(d), the proposed approach provides the more acceptable reconstructions of source positions and powers for the strong sources on the mirror and the front wheel, as well as weak ones on the back wheel.

\section{Hybrid data}

Even though our proposed approach obtains good performance on real data from wind tunnel experiments, it is not sufficient for method validation. This is because the exact acoustic source distributions on the vehicle caused by wind flow are not known beforehand. To further verify the proposed method, we use the hybrid data which composes of known synthetic sources and the real data. In order to avoid overlapping the original sources, the synthetic sources are set on the region where there are no significant sources powers. In Fig.11(a), five synthetic complex sources with different patterns are generated at $2500 \mathrm{~Hz}$, whose powers are within $[-4.5,0] \mathrm{dB}$. We expect that our proposed approach can detect both the synthetic and original source powers from the hybrid data. If these known synthetic sources are successfully recovered, the proposed approach can be able to effectively reconstruct the original acoustic sources on the vehicle surface.

For the synthetic sources, figure.11(f) shows that proposed approach successfully detects most of the source powers and patterns. For the original sources in hybrid data, the proposed approach better discovers both strong and weak sources on two wheels and rearview mirrors, as well as obtains a better noise suppression compared with mentioned methods in Fig.11(b)-(e). 


\section{Conclusions and perspectives}

In this article, we have proposed a robust super resolution approach with sparsity constraint for the acoustic imaging on the vehicle surface in wind tunnel experiments.

For the robustness to background noises, we have improved the forward model of power propagation by considering the noises at the sensors, as well as the propagation uncertainty caused by wind fraction and ground reflection in wind tunnel. For the super spatial resolution, we have adaptively estimated the sparsity parameter on source powers in the proposed Algorithm 1.

For the approach validation, we have presented performance comparisons with classical methods. The simulations have shown that proposed approach obtained the $5 \mathrm{~cm}$ super resolution compared with the beamforming resolution $31 \mathrm{~cm}$ at $2500 \mathrm{~Hz}$. It achieved $15 \mathrm{~dB}$ dynamic range of power estimations, and well detected complex sources with different patterns. The Real data results have demonstrated that proposed approach effectively reconstructed strong sources on front wheels and rear-view mirrors, as well as the weak sources on back wheels. The hybrid data experiments have furthermore confirmed the effectiveness for reconstructing the known synthetic sources and original sources in the real data. We have also shown the moderate computational cost of our approach for the acoustic imaging in wind tunnel tests.

The main drawback of proposed approach is the overweening effect existed in the compressed sensing methods. Due to the same sparsity constraint, it sometimes just obtains many unstructured or shapeless points and could not to reconstruct the true source distribution, especially when acoustic sources could not be modeled by monopoles. To overcome this limitation, it is worthwhile to investigate a hierarchical Bayesian inference with the group sparsity prior $[37,38]$ which enforces the sparsity and model the source distributions. Furthermore, we should consider the (in)coherent distributed source model that is modeled by a parametric angular cross-correlation kernel [39, 40].

\section{Acknowledgment}

The authors are deeply grateful to Dr. Jean-Luc Adam in Renault SAS for offering real data and valuable discussions on our researches.

\section{References}

[1] J. Lanslots, F. Deblauwe, K. Janssens, Selecting Sound Source Localization Techniques for Industrial Applications, Sound and Vibration 44 (6) (2010) 6-10.

[2] A. B. Nagy, Aeroacoustics research in Europe: The CEASASC report on 2010 highlights, Journal of Sound and Vibration 330 (21) (2011) 4955-4980.

[3] M. Magalhaes, R. Tenenbaum, Sound sources reconstruction techniques: A review of their evolution and new trends, Acta Acustica united with Acustica 90 (2) (2004) 199-220.

[4] J. Chen, K. Yao, R. Hudson, Source localization and beamforming, Signal Processing Magazine, IEEE 19 (2) (2002) 30-39.

[5] B. Van Veen, K. Buckley, Beamforming: A versatile approach to spatial filtering, ASSP Magazine, IEEE 5 (2) (1988) 4-24.

[6] R. O. Schmidt, Multiple emitter location and signal parameter estimation, IEEE Transactions on Antennas and Propagation 34 (1986) 276-280.

[7] P. Sijtsma, CLEAN based on spatial source coherence, International Journal of Aeroacoustics 6 (4) (2007) 357-374.

[8] T. Brooks, W. Humphreys, A Deconvolution Approach for the Mapping of Acoustic Sources (DAMAS) determined from phased microphone arrays, Journal of Sound and Vibration 294 (4-5) (2006) 856-879.

[9] T. Yardibi, J. Li, P. Stoica, L. Cattafesta III, Sparsity constrained deconvolution approaches for acoustic source mapping, The Journal of the Acoustical Society of America 123 (5) (2008) $2631-42$.

[10] T. Yardibi, J. Li, P. Stoica, N. S. Zawodny, L. N. Cattafesta, A covariance fitting approach for correlated acoustic source mapping, Journal of The Acoustical Society of America 127 (5) (2010) 2920-31.

[11] D. Blacodon, Spectral estimation method for noisy data using a noise reference, Applied Acoustics 72 (1) (2011) 11-21.

[12] D. Blacodon, Array processing for noisy data: Application for open and closed wind tunnels, AIAA journal 49 (1) (2011) 5566.

[13] D. Malioutov, M. Çetin, A. Willsky, A sparse signal reconstruction perspective for source localization with sensor arrays, IEEE Transactions on Signal Processing 53 (8) (2005) 3010-3022.

[14] N. P. Galatsanos, A. K. Katsaggelos, Methords for choosing the regularization parameters and estimating the noise variance 
in image restoration and thier relation, IEEE Transactions on Image Processing 1 (3) (1992) 332-336.

[15] T. Suzuki, L1 generalized inverse beam-forming algorithm resolving coherent/incoherent, distributed and multipole sources, Journal of Sound and Vibration 330 (24) (2011) 5835 - 5851.

[16] A. Massa, G. Oliveri, Bayesian compressive sampling for pattern synthesis with maximally sparse non-uniform linear arrays, IEEE Transactions on Antennas and Propagation 59 (10) (2011) $467-681$.

[17] G. Oliveri, P. Rocca, A. Massa, A bayesian-compressivesampling-based inversion for imaging sparse scatterers, IEEE Transactions on Geoscience and Remote Sensing (99) (2011) 3993-4006.

[18] J. Antoni, A Bayesian approach to sound source reconstruction: optimal basis, regularization, and focusing, The Journal of the Acoustical Society of America 131 (2012) 2873-2890.

[19] N. Chu, A. Mohammad-Djafari, J. Picheral, Robust Bayesian super-resolution approach via sparsity enforcing a priori for near-field aeroacoustic source imaging, Journal of Sound and Vibration 332 (18) (2013) 4369-4389.

[20] Y. Wang, J. Li, P. Stoica, M. Sheplak, T. Nishida, Wideband RELAX and wideband CLEAN for aeroacoustic imaging, Journal of Acoustical Society of America 115 (2) (2004) 757-767.

[21] E. Sarradj, A fast signal subspace approach for the determination of absolute levels from phased microphone array measurements, Journal of Sound and Vibration 329 (9) (2010) 15531569 .

[22] P. Simard, J. Antoni, Acoustic source identification: Experimenting the 11 minimization approach, Applied Acoustics 74 (7) (2013) $974-986$.

[23] J.-L. Adam, D. Ricot, C. Lambourg, A. Menoret, Correlated Beamforming Method for Relevant Aeroacoustic Sources Identification, in: SAE 2009 Noise and Vibration Conference and Exhibition, SAE, St. Charles, Illinois, United States, 20-23 May 2009, pp. 2009-01-2234.

[24] Q. Leclère, Acoustic imaging using under-determined inverse approaches: Frequency limitations and optimal regularization, Journal of Sound and Vibration 321 (3-5) (2009) 605-619.

[25] R. Tibshirani, Regression shrinkage and selection via the LASSO, Journal of the Royal Statistical Society. Series B (Methodological) 58 (1) (1996) 267-288.

[26] S. Chen, D. Donoho, M. Saunders, Atomic decomposition by basis pursuit, SIAM journal on scientific computing 20 (1) (1999) $33-61$.

[27] J. Fuchs, Multipath time-delay detection and estimation, IEEE Transactions on Signal Processing 47 (1) (1999) 237-243.

[28] Y. Kim, P. Nelson, Optimal regularisation for acoustic source reconstruction by inverse methods, Journal of sound and vibra- tion 275 (3-5) (2004) 463-487.

[29] B. M. Radich, K. M. Buckley, Single-snapshot DOA estimation and source number detection, IEEE Signal Processing Letters 4 (4) (1997) 109-111.

[30] N. Chu, J. Picheral, A. Mohammad-Djafari, A robust superresolution approach with sparsity constraint for near-field wideband acoustic imaging, in: IEEE International Symposium on Signal Processing and Information Technology, Bilbao, Spain, Dec.14-17,2011, pp. 310-315.

[31] D. L. Donoho, M. Elad, V. N. Temlyakov, Stable recovery of sparse overcomplete representations in the presence of noise, IEEE Transactions on Information Theory 52 (1) (2006) 6-18.

[32] E. Candes, J. Romberg, Sparsity and incoherence in compressive sampling, Inverse problems 23 (3) (2007) 969.

[33] Z. Ben-Haim, Y. C. Eldar, M. Elad, Coherence-based performance guarantees for estimating a sparse vector under random noise, IEEE Transactions on Signal Processing 58 (10) (2010) 5030-43.

[34] J. Sturm, Using sedumi 1.02, a matlab toolbox for optimization over symmetric cones, Optimization methods and software 11 (1-4) (1999) 625-653.

[35] A. Menoret, N. Gorilliot, J.-L. Adam, Acoustic imaging in wind tunnel S2A, in: 10th Acoustics conference (ACOUSTICS2010), Lyon, France, 2010.

[36] C. E. Kassis, J. Picheral, C. Mokbel, Advantages of nonuniform arrays using root-music, Signal Processing 90(2) (2010) 689695.

[37] J. Huang, T. Zhang, The benefit of group sparsity, The Annals of Statistics 38 (4) (2010) 1978-2004.

[38] R. Jenatton, J.-Y. Audibert, F. Bach, Structured variable selection with sparsity-inducing norms, The Journal of Machine Learning Research 12 (2011) 2777-2824.

[39] S. Shahbazpanahi, S. Valaee, A. B. Gershman, A covariance fitting approach to parametric localization of multiple incoherently distributed sources, IEEE Transactions on Signal Processing 52 (3) (2004) 592-600.

[40] J. Lee, I. Song, H. Kwon, S. Ro Lee, Low-complexity estimation of 2D DOA for coherently distributed sources, Signal processing 83 (8) (2003) 1789-1802. 


\section{List of Figures}

1 Illustration of acoustic propagation in the wind tunnel $[19]$. . . . . . . . . . . . . 3

2 Simulation on monopole sources with $14 \mathrm{~dB}$ power dynamic range at $2500 \mathrm{~Hz}, \sigma^{2}=0.86$, $\mathrm{SNR}=0 \mathrm{~dB}$ and $15 \mathrm{~dB}$ display: (a) Monopole sources (b) CBF (c) DAMAS with 5000 iterations (5000i) (d) CLEAN (e) SC-DAMAS (f) DR-DAMAS (5000i) (g) CMF and (h) Proposed SC-RDAMAS . . . . . . . . .

3 Performance comparison for relative errors of power image reconstruction $\delta_{2}$ versus SNR $[-6,18] \mathrm{dB}$ on simulations at $2500 \mathrm{~Hz}$. . . . . 10

4 Performance comparison for relative errors of power image reconstruction $\delta_{2}$ versus [1600, $2600] \mathrm{Hz}$ on simulations at $\mathrm{SNR}=3 \mathrm{~dB} . \quad \ldots \quad 10$

5 Sparsity parameter influence caused by estimated source number $\hat{K}$ versus power image reconstruction error $\delta_{2}$ between the SCDAMAS and proposed SC-RDAMAS at 0dB and $2500 \mathrm{~Hz} \ldots \ldots \ldots \ldots \ldots \ldots$

6 Wind tunnel S2A [35] in France. . . . . . . 12

7 Acoustic imaging of real data on the whole car side at 2500Hz: (a) CBF (b) DAMAS (5000i) (c) DR-DAMAS (5000i) (d) CLEAN (e) SC-DAMAS and (f) Proposed SC-RDAMAS. 13

9 Sparsity parameter influence of proposed SCRDAMAS on real data at 2500Hz: (a) Underestimated $\hat{\beta}$ (b) Over-estimated $\hat{\beta} . \ldots .13$

8 Acoustic imaging of rear-view mirror at $2500 \mathrm{~Hz}$ :

(a) CBF (b) DAMAS (5000i) (c) DR-DAMAS

(5000i) (d) CMF (e) SC-DAMAS and (f) Proposed SC-RDAMAS . . . . . . . . . 19

10 Wide-band data over $[2400,2600] \mathrm{Hz}$ : (a) DAMAS

(b) CLEAN (c) SC-DAMAS and (d) Proposed SC-RDAMAS ............. . 19
11 Acoustic imaging of hybrid data on the whole car side at 2500Hz: (a) Synthetic sources (b) CBF (c) DAMAS (5000i) (d) CLEAN

(e) SC-DAMAS and (f) Proposed SC-RDAMAS. 19 


\section{List of Tables}

1 Power estimations of 4 monopole sources by average power estimation error $\overline{\Delta x^{*}}$, relative errors of power image reconstruction $\delta_{2}$ and estimated noise power $\hat{\sigma}^{2}$ at $2500 \mathrm{~Hz}$, $\mathrm{SNR}=0 \mathrm{~dB}$, simulated $\sigma^{2}=0.86$; '-' means unavailable. . . . . . . . . . . .

2 Position estimations of 4 monopole sources by averaged position errors $\overline{\Delta p^{*}}$ at $2500 \mathrm{~Hz}$, $\mathrm{SNR}=0 \mathrm{~dB}$; '-' means unavailable. . . . . . . 10

3 Power estimations of the complex monopole source on the center of image by power estimation error $\overline{\Delta x^{*}}$ at $2500 \mathrm{~Hz}, \mathrm{SNR}=0 \mathrm{~dB}$; '-' means unavailable. . . . . . . . . . . . 10

4 Computational cost for treating whole car: image $30 \times 100$ pixels, at $2500 \mathrm{~Hz}$, based on CPU:3.33GHz, '-' means unavailable. . . . . 13 
(a)

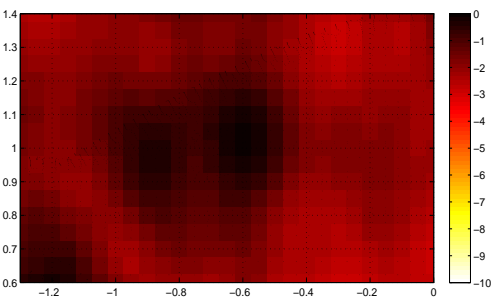

(d)

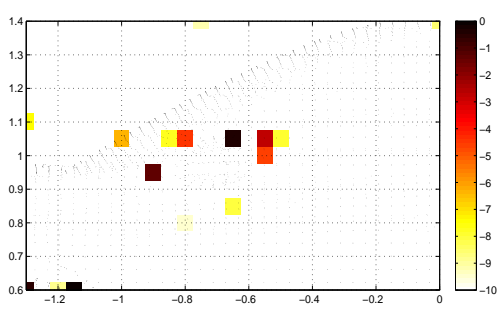

(b)

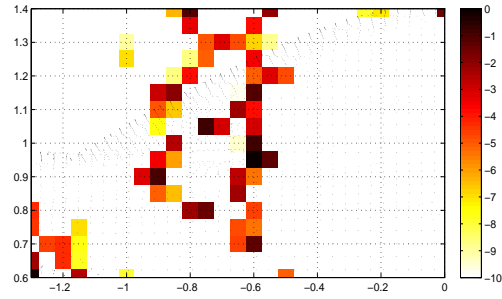

(e)

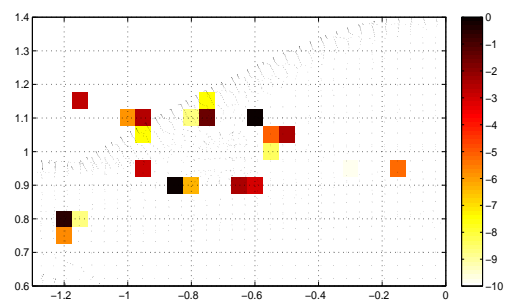

(c)

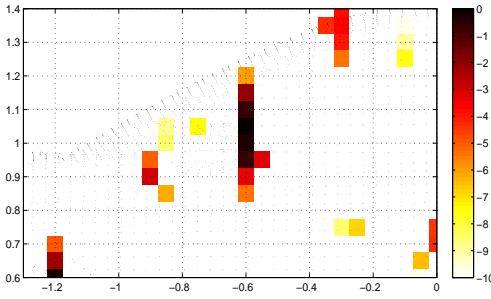

(f)

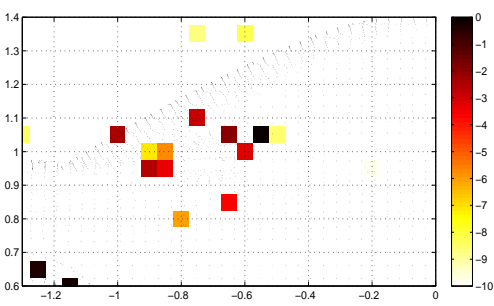

Figure 8: Acoustic imaging of rear-view mirror at 2500Hz: (a) CBF (b) DAMAS (5000i) (c) DR-DAMAS (5000i) (d) CMF (e) SC-DAMAS and (f) Proposed SC-RDAMAS

(a)

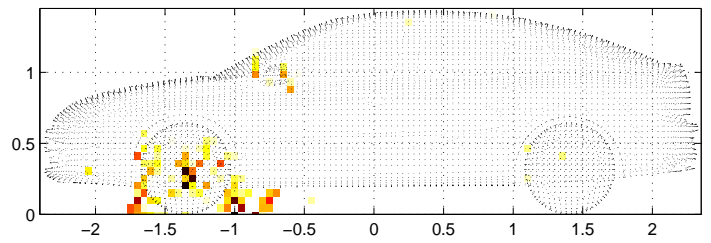

(c)

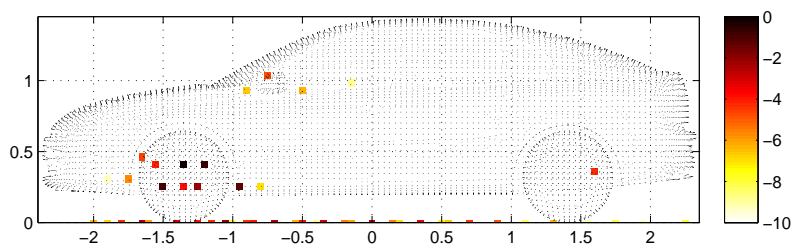

(b)

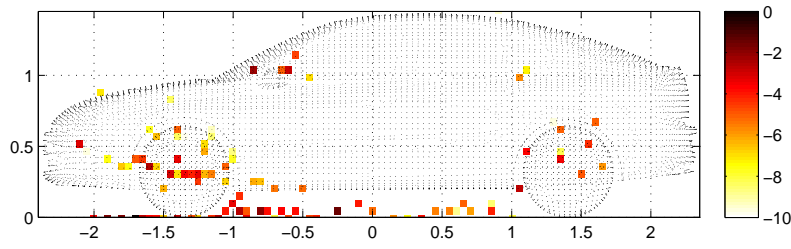

(d)

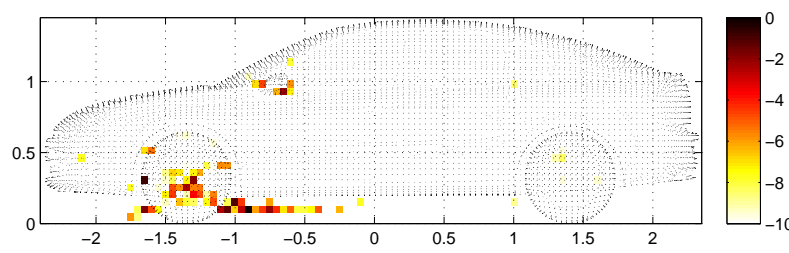

Figure 10: Wide-band data over [2400,2600]Hz: (a) DAMAS (b) CLEAN (c) SC-DAMAS and (d) Proposed SC-RDAMAS

(a)

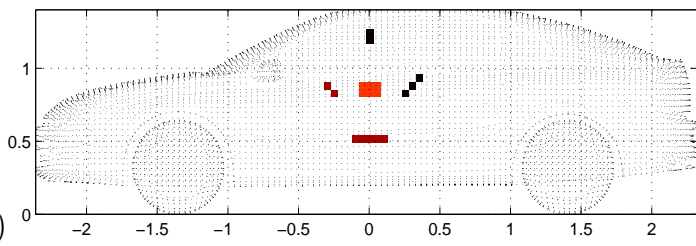

(c)

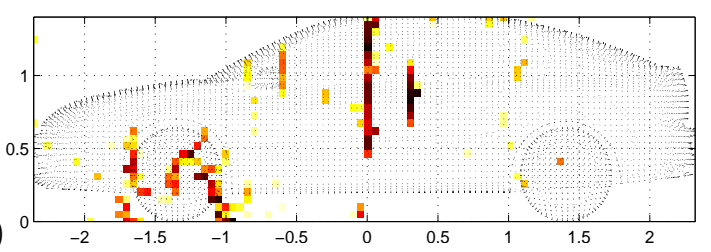

(e)

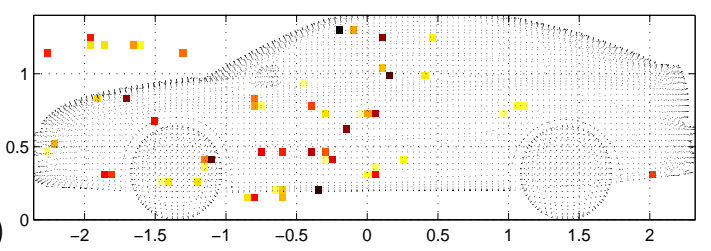

(b)
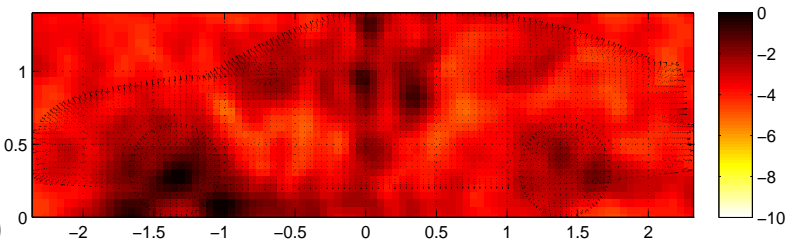

(d)

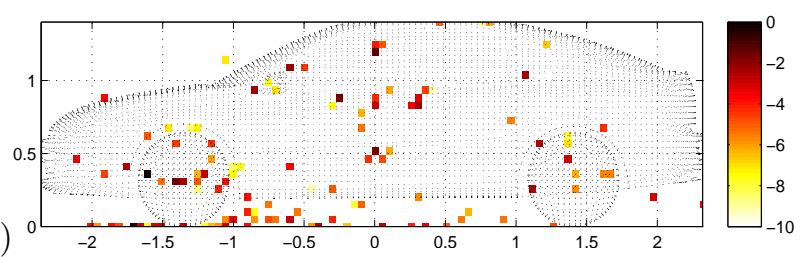

(f)

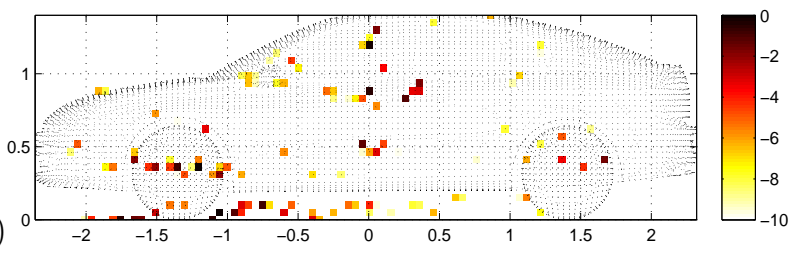

Figure 11: Acoustic imaging of hybrid data on the whole car side at 2500Hz: (a) Synthetic sources (b) CBF (c) DAMAS (5000i) (d) CLEAN (e) SC-DAMAS and (f) Proposed SC-RDAMAS. 